\title{
Genome and time-of-day transcriptome of Wolffia australiana link morphological minimization with gene loss and less growth control
}

\author{
Todd P. Michael, ${ }^{1}$ Evan Ernst, ${ }^{2,3,11}$ Nolan Hartwick, ${ }^{1,11}$ Philomena Chu, ${ }^{4,12}$ \\ Douglas Bryant, ${ }^{5,13}$ Sarah Gilbert, ${ }^{4,14}$ Stefan Ortleb, $^{6}$ Erin L. Baggs, ${ }^{7}$ K. Sowjanya Sree, ${ }^{8}$ \\ Klaus J. Appenroth, ${ }^{9}$ Joerg Fuchs, ${ }^{6}$ Florian Jupe, ${ }^{1,15}$ Justin P. Sandoval, ${ }^{1}$ \\ Ksenia V. Krasileva, ${ }^{7}$ Ljudmylla Borisjuk, ${ }^{6}$ Todd C. Mockler, ${ }^{5}$ Joseph R. Ecker, ${ }^{1,10}$ \\ Robert A. Martienssen, ${ }^{2,3}$ and Eric Lam ${ }^{4}$ \\ ${ }^{1}$ Plant Molecular and Cellular Biology Laboratory, The Salk Institute for Biological Studies, La Jolla, California 92037, USA; ${ }^{2}$ Cold \\ Spring Harbor Laboratory, Cold Spring Harbor, New York 11724, USA; ${ }^{3}$ Howard Hughes Medical Institute, Cold Spring Harbor \\ Laboratory, Cold Spring Harbor, New York 11724, USA; ${ }^{4}$ Department of Plant Biology, Rutgers, The State University of New Jersey, \\ New Brunswick, New Jersey 08901, USA; ${ }^{5}$ Donald Danforth Plant Science Center, St. Louis, Missouri 631 32, USA; ${ }^{6}$ Leibniz Institute of \\ Plant Genetics and Crop Plant Research (IPK), Gatersleben 06466, Germany; ${ }^{7}$ Department of Plant and Microbial Biology, University \\ of California, Berkeley, Berkeley, California 94720, USA; ${ }^{8}$ Department of Environmental Science, Central University of Kerala, Periye, \\ Kerala 671316, India; ${ }^{9}$ Friedrich Schiller University of Jena, Jena 07737, Germany; ${ }^{10}$ Howard Hughes Medical Institute, The Salk \\ Institute for Biological Studies, La Jolla, California 92037, USA
}

Rootless plants in the genus Wolffia are some of the fastest growing known plants on Earth. Wolffia have a reduced body plan, primarily multiplying through a budding type of asexual reproduction. Here, we generated draft reference genomes for Wolffia australiana (Benth.) Hartog \& Plas, which has the smallest genome size in the genus at $357 \mathrm{Mb}$ and has a reduced set of predicted protein-coding genes at about 15,000. Comparison between multiple high-quality draft genome sequences from W. australiana clones confirmed loss of several hundred genes that are highly conserved among flowering plants, including genes involved in root developmental and light signaling pathways. Wolffia has also lost most of the conserved nucleotidebinding leucine-rich repeat (NLR) genes that are known to be involved in innate immunity, as well as those involved in terpene biosynthesis, while having a significant overrepresentation of genes in the sphingolipid pathways that may signify an alternative defense system. Diurnal expression analysis revealed that only $13 \%$ of Wolffia genes are expressed in a time-of-day (TOD) fashion, which is less than the typical $\sim 40 \%$ found in several model plants under the same condition. In contrast to the model plants Arabidopsis and rice, many of the pathways associated with multicellular and developmental processes are not under TOD control in W. australiana, where genes that cycle the conditions tested predominantly have carbon processing and chloroplast-related functions. The Wolffia genome and TOD expression data set thus provide insight into the interplay between a streamlined plant body plan and optimized growth.

[Supplemental material is available for this article.]

Wolffia has the distinction of being the duckweed genus with the smallest (Wolffia angusta) as well as the fastest growing (Wolffia microscopica) species of known flowering plants (Sree et al. 2015b). Plants belonging to this genus are highly reduced in their morphology and anatomy, lacking roots and containing only the

\footnotetext{
${ }^{11}$ These authors contributed equally to this work. Present addresses: ${ }^{12}$ Department of Energy and Process Engineering, Norwegian University of Science and Technology, Trondheim NO7491, Norway; ${ }^{13}$ NewLeaf Symbiotics, BRDG Park, St. Louis, MO 63132, USA; ${ }^{4}$ Department of Biology, University of North Carolina, Chapel Hill, NC 27599, USA; ${ }^{15}$ Bayer Crop Science, Chesterfield, MO 63017, USA

Corresponding authors: tmichael@salk.edu, eric.lam@rutgers.edu Article published online before print. Article, supplemental material, and publication date are at http://www.genome.org/cgi/doi/10.1101/gr.266429.120. Freely available online through the Genome Research Open Access option.
}

green floating frond, which is essentially a fused leaf and stem without any vasculature (Fig. 1).

Wolffia typically measure only a few millimeters to less than a millimeter in size (Landolt 1986) and grow as colonies of two individuals, one mother frond budding and giving rise to one or more daughter fronds (Fig. 1A). Anatomically, however, at least four different generations of plants (total of 10-14 individuals) can be found within one colony, highlighting their adaptive preparedness for fast vegetative multiplication (Fig. 1C; Bernard et al. 1990; Lemon and Posluszny 2000; Sree et al. 2015a; Supplemental Movie S1).

Wolffia is part of a family of aquatic non-grass monocots known as duckweeds (Lemnaceae) (Fig. 1B). There are five genera

() 2021 Michael et al. This article, published in Genome Research, is available under a Creative Commons License (Attribution-NonCommercial 4.0 International), as described at http://creativecommons.org/licenses/by-nc/4.0/. 
A

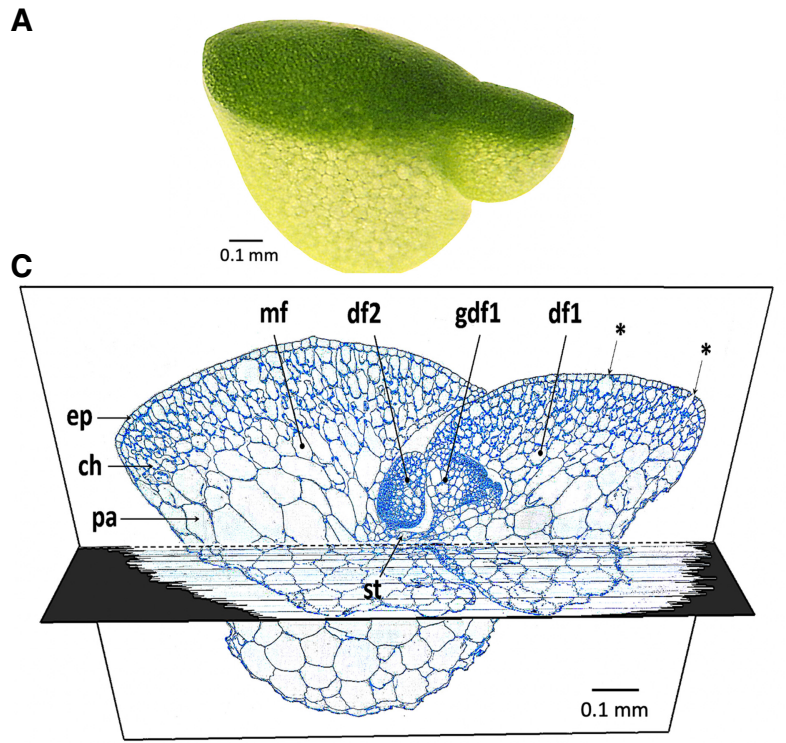

B

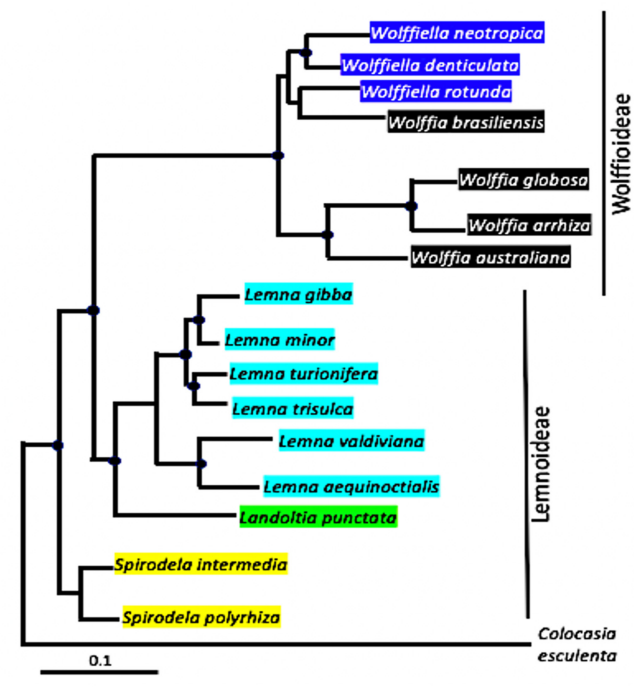

Figure 1. Wolffia is a simple plant with a limited number of cells and structures. ( $A$ ) Brightfield image of wa8730. A video with time tracking of the asexual propagation of wa7733 in culture can be found in the Supplemental Material. (B) Phylogenetic relationship between representative species from the five genera of duckweed. Each color represents a distinct genus. Modified from Borisjuk et al. (2015). (C) Cross section (1- $\mu$ m-thick section) of W. australiana stained with methylene blue: (mf) Mother frond; (df) daughter frond; (gdf) grand-daughter frond; (ep) epidermal cells; (ch) chlorenchyma cells; (pa) parenchyma cells; asterisks indicate some of the stomata; (st) stipe tissue.

of duckweed (Spirodela, Landoltia, Lemna, Wolffiella, and Wolffia), and their genome sizes span an order of magnitude (Wang et al. 2011; Bog et al. 2015; Hoang et al. 2019). The Greater Duckweed, Spirodela polyrhiza, which is the most basal with the largest body size and most complex organization, has the smallest genome at $158 \mathrm{Mb}$, whereas in Wolffia, the most derived genus, genome sizes range from $W$. australiana at $357 \mathrm{Mb}$ to $W$. arrhiza at $1881 \mathrm{Mb}$. $S$. polyrhiza (sp7498) was the first duckweed genome to be sequenced, which revealed a reduced set of protein-coding genes at 19,623 that are conserved across flowering plants (Wang et al. 2014; An et al. 2019). A chromosome-resolved genome for a second accession sp9509 revealed that in addition to a reduced gene set, the Spirodela genome has low intraspecific variance, highly reduced ribosomal arrays, and minimal cytosine methylation, consistent with the genome being specialized for growth (Michael et al. 2017; Hoang et al. 2018).

Green organisms from algae to higher plants partition their biology to coincide with the light-dark cycle, which enhances their ability to anticipate changing conditions (Green et al. 2002; Michael et al. 2003; Dodd et al. 2005; Ferrari et al. 2019). In the model plant Arabidopsis thaliana, as much as $90 \%$ of its genes are expressed, or phased, to a specific TOD to optimize growth, and this global transcriptional regulation is conserved across higher plants (Michael et al. 2008a,b; Filichkin et al. 2011). Because Wolffia is the fastest growing angiosperm known to date with a doubling time of as little as a day, we wanted to understand what special adaptations in the genome enabled this rapid growth.

\section{Results}

\section{Wolffia genome}

We performed whole-genome sequencing and time-of-day (TOD) expression profiling of Wolffia australiana, the species with the smallest reported genome of the Wolffia genus, with an estimated size of $375 \mathrm{Mb}$ for accession wa8730 and $357 \mathrm{Mb}$ for accession wa7733 (Wang et al. 2011). Both accessions are from Australia with wa7733 from Mount Lofty Range, Torrens Gorge, in South Australia $\left(34^{\circ} \mathrm{S}, 138^{\circ} \mathrm{E}\right)$; wa8730 is from Singleton, Doughboy Hollow, New South Wales $\left(32^{\circ} \mathrm{S}, 151^{\circ} \mathrm{E}\right)$. These two $W$. australiana accessions (wa7733 and wa8730) have doubling times of 1.56 and 1.66 d, respectively (Supplemental Table S1; Supplemental Fig. S1), consistent with the previous measurement of $1.39 \mathrm{~d}$ for this species (Sree et al. 2015b). We sequenced and de novo assembled wa7733 and wa8730 using Pacific Biosciences (PacBio) single molecule real-time sequencing (SMRT). We also generated BioNano optical maps to correct contigs and help initial scaffolding of the assemblies (Supplemental Table S2), which resulted in final assemblies of 393 and $354 \mathrm{Mb}$, with longest scaffolds of 5.3 and $1.7 \mathrm{Mb}$, and scaffold N50 lengths of 836 and $109 \mathrm{~kb}$ for wa7733 and wa8730, respectively (Table 1 ). The assembled genome sizes are consistent with those predicted by $k$-mer $(k=19)$ frequency analysis (Supplemental Fig. S2), but smaller than predicted by our new flow cytometry estimates possibly reflecting missing high copy number repeat sequence (centromeres) in our assemblies (Supplemental Table S3; Supplemental Materials).

We checked the completeness of these assemblies by mapping 1780 high confidence Sanger sequenced $W$. australiana cDNA clone sequences deposited at GenBank by the Waksman Student Scholar Program (WSSP). We found that 100\% and $99.7 \%$ of the cDNAs mapped to the wa7733 and wa8730 genomes, respectively. Also, the PacBio reads used for the assembly and the Illumina reads used for polishing had a $>95 \%$ mapping rate to their respective assemblies, consistent with the lack of contamination (bacterial) in the sequencing data and the completeness of the assemblies (Table 1). We were also able to identify megabases of putative centromere arrays in both assemblies with three prominent base unit sizes of 126, 167, and 250 bp (Supplemental Fig. S3).

Repeat sequence is a dominant driver of genome size differences in plants (Michael and VanBuren 2020). We looked at

\section{Genome Research}

www.genome.org 
Table 1. Genome assembly statistics

\begin{tabular}{|c|c|c|c|c|}
\hline & wa7733 & wa8730 & sp9509 & sp7498 \\
\hline Genome size estimate flow cytometry (Mb) & 441 & 432 & 157 & 157 \\
\hline Genome size estimate $k$-mer $(\mathrm{Mb})$ & 343 & 342 & 164 & 185 \\
\hline Genome final assembly (bp) & $393,842,592$ & $354,558,446$ & $138,592,155$ & $145,274,398$ \\
\hline Contig (\#) & 2578 & 5250 & 20 & 21 \\
\hline Genome contig assembly size (bp) & $359,766,217$ & $337,899,876$ & NA & NA \\
\hline Longest contig (bp) & $1,664,978$ & 679,034 & NA & NA \\
\hline N50 contig length (bp) & 256,298 & 102,418 & NA & NA \\
\hline L50 contig (\#) & 404 & 1000 & NA & NA \\
\hline Longest scaffold (bp) & $5,333,369$ & $1,714,878$ & $11,560,055$ & $12,728,324$ \\
\hline N50 scaffold length (bp) & 836,551 & 109,493 & $7,949,387$ & $8,107,549$ \\
\hline L50 scaffold (\#) & 122 & 753 & 8 & 8 \\
\hline BUSCO complete scaffolds (\%) & 69 & 70 & 79 & 78 \\
\hline Full-length LTRs (\#) & 2510 & 1892 & 801 & 567 \\
\hline Masked (\%) & 50 & 50 & 26 & 25 \\
\hline Solo:intact LTR ratio & 11 & 14 & 8 & NA \\
\hline Protein-coding genes (\#) & 15,312 & 14,324 & 17,510 & 17,057 \\
\hline cDNA mapping (\%) & 100 & 99.7 & NA & NA \\
\hline Coverage PacBio (fold) & 91 & 45 & NA & NA \\
\hline Coverage llumina (fold) & 80 & 68 & NA & NA \\
\hline Mapping PacBio (\%) & 98 & 96 & NA & NA \\
\hline Mapping Illumina (\%) & 98 & 95 & NA & NA \\
\hline
\end{tabular}

(NA) Not available.

ribosomal DNA (rDNA) and long terminal repeat retrotransposon (LTR-RT) content in the $W$. australiana genomes to understand whether, as in Spirodela, the smallest genome in this genus (Wang et al. 2011; Hoang et al. 2019), Wolffia has also purged repetitive sequences. We found approximately 200 copies of the rDNA array in the $W$. australiana genomes, double that of Spirodela but still half the number found in Arabidopsis (Supplemental Table S4). Next, we identified full-length, intact LTR-RT across both genomes and found 2510 and 1892 for wa7733 and wa8730, respectively, which was three times more than Spirodela (Table 1). Retroelements (solo+intact) made up $>50 \%$ of both of the Wolffia genomes, which is twice the repeat content found in Spirodela (Michael et al. 2017). It has been shown that the ratio between intact LTR-RTs and solo LTRs (solo:intact), which are left over after illegitimate recombination, is a proxy for how actively the genome is purging proliferating LTR-RTs (Devos et al. 2002). Although Spirodela has a high solo:intact ratio of 8 (Michael et al. 2017), Wolffia has an even higher ratio at 11-14 in wa7733 and wa8730, respectively (Table 1), consistent with it more actively purging its TEs leading to a much smaller sized genome than other Wolffia species (Wang et al. 2011).

The two draft Wolffia genomes are highly collinear with one another at the gene level, but there are examples of structural changes and gene loss and gain (Fig. 2A; Supplemental Fig. S4).
A

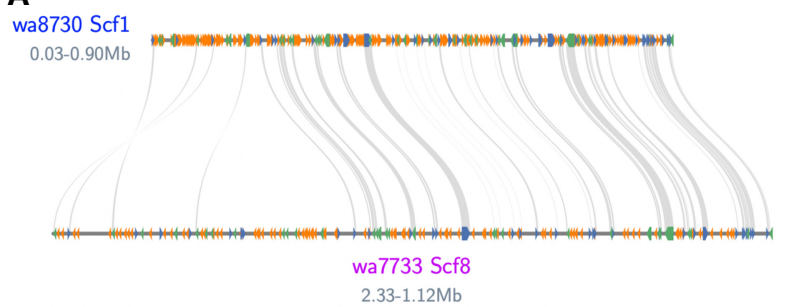

B

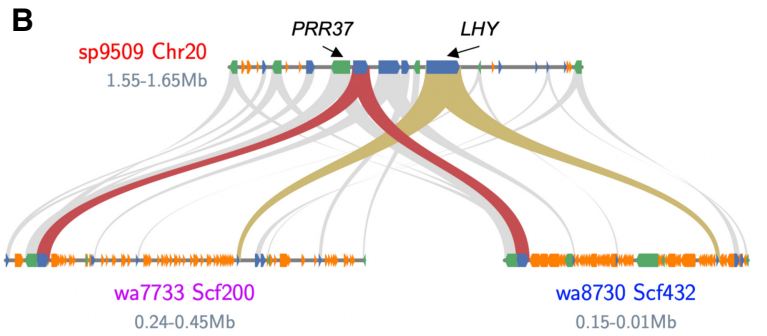

C

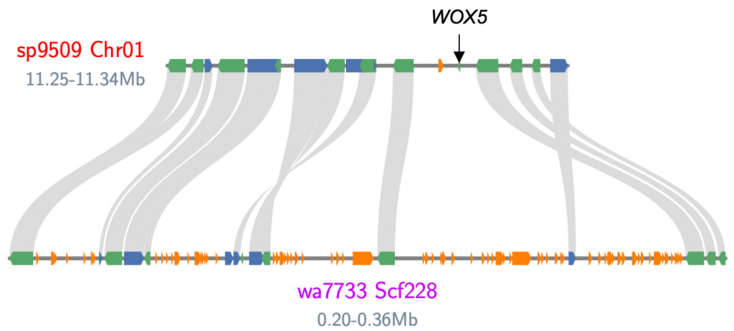

D

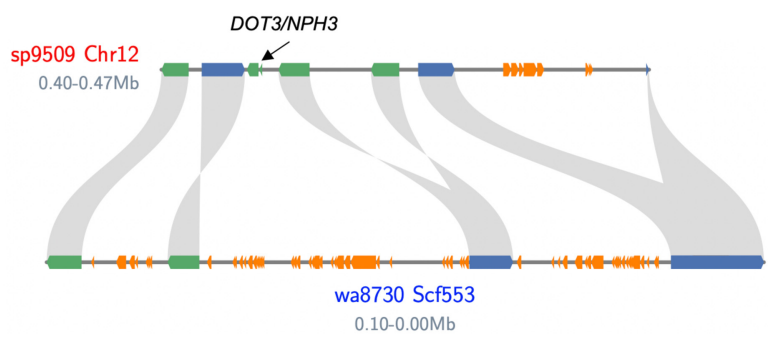

Figure 2. The Wolffia genome is collinear with Spirodela with bloating and loss of genes owing to transposable elements (TEs). ( $A$ ) wa8730 scaffold 1 (scf1) is collinear with wa7733 scf8. (B) Conserved core circadian clock genes PRR37 (red ribbon) and LHY (gold ribbon) on sp9509 Chromosome 20 (Chr 20) are collinear in wa8730 scf200 and wa7733 scf432. (C) WOX5 is lost in Wolffia compared to Spirodela owing to TE insertions (yellow boxes). (D) DOT3/NPH3 loci in Spirodela lost in Wolffia owing to LTR-RT insertions. Gray lines represent syntenic connections. Green and blue boxes are forward and reverse representation for genes, respectively. Orange boxes are TEs. 
Most of the structural variations are associated with insertion/deletions (indels) between 50 and 500 bp (Supplemental Fig. S5). In addition, both Wolffia genomes are largely collinear with Spirodela (Fig. 2B-D; Supplemental Fig. S6). One specific example is in the evolutionarily conserved linkage between core circadian clock genes LATE ELONGATED HYPOCOTYL (LHY) and PSEUDO RESPONSE REGULATOR 37 (PRR37) on Chr 20 of the sp9509 genome that is found as far back as bryophytes and shared across other dicots and non-grass monocots (Fig. 2B). Although the genic regions of the Wolffia genomes are mostly collinear with those of the Spirodela genomes, the intergenic and repeat sequences are expanded in the Wolffia genomes, explaining its larger genome sizes (Fig. 2C,D; Supplemental Fig. S6).

\section{Comparative BUSCO analysis reveals genes missing in Wolffia}

As an additional check of genome assembly completeness, we leveraged Benchmarking Universal Single-Copy Orthologs (BUSCO) (Simão et al. 2015), which searches the genome for near-universal single-copy orthologs to establish how much of the gene space has been properly assembled. We found that wa7733 and wa8730 contained $69 \%$ and $70 \%$ complete BUSCOs, respectively (Table 1; Supplemental Table S5). The BUSCO scores for percent complete were lower than we had expected based on the contiguity (N50 length and longest contigs) as well as the cDNA and read mapping results (Table 1). Therefore, we compared the missing BUSCO genes between the two Wolffia assemblies and the two previously published highquality Spirodela genomes, sp9509 and sp7498 (Wang et al. 2014; Michael et al. 2017; Hoang et al. 2018) and found that most of these "missing genes" were significantly shared across the four duckweed assemblies (Supplemental Fig. S7).

It is possible that some of the missing BUSCO genes represent important genes for land plants that are lost in the Wolffia genome owing to its minimal body plan and life cycle. Of the 762 and 731 missing BUSCO genes in wa7733 and wa8730, respectively, 574 (76\%-79\%) genes were shared between them (Supplemental Fig. S7; Supplemental Table S6). Two specific groups of genes emerged from this list of 574 genes: first, genes that are involved with root initiation and development, and second, genes associated with cell fate and gravitropism. Of particular interest was the loss of WUSCHEL RELATED HOMEOBOX 5 (WOX5), which is a homeodomain transcription factor responsible for root stem cell maintenance in the meristem (Sarkar et al. 2007). Because Spirodela has roots, we indeed found a likely ortholog of WOX5, but the Wolffia version of this gene has been lost and in its expected genomic location are LTR-RT, suggesting a mechanism by which this gene may have been lost (Fig. 2C). Moreover, Wolffia is also missing TOPLESS RELATED 2 (TPL2), which through chromatin-mediated repression specifies where stem cell daughters will exit stem cell fate in Arabidopsis (Pi et al. 2015). Wolffia is missing other genes associated with stem cell fate (BLISTER, FEZ) (Willemsen et al. 2008; Schatlowski et al. 2010), mediator complex (MED3, MED9, MED33) (Mathur et al. 2011), and gravitropism (LAZY1) (Yoshihara and Spalding 2017), which together are consistent with modified signaling and transcriptional cascades for a rootless, organless plant.

\section{Wolffia has a reduced set of core plant genes}

We predicted protein-coding gene structures and performed gene family analysis on the two Wolffia genomes and the two published Spirodela genomes using a standardized pipeline to ensure consis- tency (Supplemental Material). Similar to Spirodela, we found that Wolffia also had reduced gene sets in spite of their larger genome sizes. The wa7733 and wa8730 genome assemblies contain 15,312 and 14,324 predicted protein-coding genes, respectively (Table 1), which is several thousand genes less than that found in the Spirodela genome (Wang et al. 2014; Michael et al. 2017). We compared predicted proteomes from the four duckweed genomes to 28 proteomes from complete genomes spanning algae, non-seed plants, monocots, and dicots (PLAZA v4 monocots) (Van Bel et al. 2018). A multidimensional scaling (MDS) plot of the orthogroups (OGs) placed Wolffia and Spirodela next to the non-grass monocots and close to the grasses consistent with their evolutionary position (Supplemental Fig. S8; One Thousand Plant Transcriptomes Initiative 2019). Duckweeds were nested between dicot crops, basal plants, and a tree, and distant from non-seed plants and algae, consistent with having a core set of higher plant proteins (Wang et al. 2014).

Almost all of the Spirodela and Wolffia proteins were found in OGs (93\%-98) (Supplemental Table S7), with the majority (33\%$41 \%$ ) having only one protein per OG (Supplemental Fig. S9), which means that duckweed has a core set of proteins with few retained paralogs (expanded families). In contrast, species like Arabidopsis, rice, Brachypodium, and maize have almost $20 \%$ of their proteins in OGs with more than 10 paralogs (Supplemental Fig. S9). There were 408 and 635 Wolffia and Spirodela specific OGs, respectively (as compared to rice, Arabidopsis, Zostera, and banana), and 77 OGs exclusive to both (Fig. 3A). The OGs unique to Wolffia can be summarized into the significant Gene Ontology (GO) categories (FDR < 0.05) of sphingolipid biosynthesis, photomorphogenesis, wax biosynthetic, and cysteine-type endopeptidase (Fig. 3B; Supplemental Table S8); we found an overlapping set of duckweed-specific significant GO terms (Fig. 3D). We took a look at the genes that made up these Wolffia unique OGs using our annotation and found that they are associated with cell wall architecture (Fasciclin-like arabinogalactan proteins) (Johnson et al. 2011), environment-specific expression orchestration (nuclear transcription factor Y) (Zhao et al. 2016), flowering time (Casein kinase 1-like HD16) (Hori et al. 2013), and sphingolipids (Fig. 3B; Supplemental Table S9; Huby et al. 2020).

In contrast, the significant OGs (FDR $>0.05$ ) missing in Wolffia included cell wall, flavonoid biosynthesis, protein phosphorylation, immune response, and terpene biosynthesis (Fig. 3C; Supplemental Table S10). Looking at specific genes with known function suggests there is a loss of OGs involved in meristem development (FAF, BON1, SCL3/11, POLAR, PSD, SZ1), chromatin (MED4, POLD3), and light signaling (BBX12, DOT3/NPH3, GBF4) (Supplemental Table S10). Another OG that is completely missing in Wolffia but has a large family in Arabidopsis $(n=11)$, rice $(n=9)$ and most other land plants, is the CASPARIAN STRIP MEMBRANE DOMAIN PROTEIN (CASPS) family (Roppolo et al. 2014).

Some of the most significant missing GO terms were in the terpene biosynthesis pathway, whereas the most significant Wolffia-specific as well as duckweed-specific GOs were in the sphingolipid-related pathways (Fig. 3B,D). Because both terpenes and sphingolipids may play a predominant role in plant defense (Singh and Sharma 2015; Huby et al. 2020), it is possible that Wolffia has traded the terpene pathway for sphingolipids or that the aquatic environment favors the latter. Related to genes involved in defense, one of the most conserved gene families in plants is the nucleotide-binding leucine-rich repeat (NLR) superfamily encoding hundreds of disease resistance (R) genes that

\section{Genome Research}

www.genome.org 


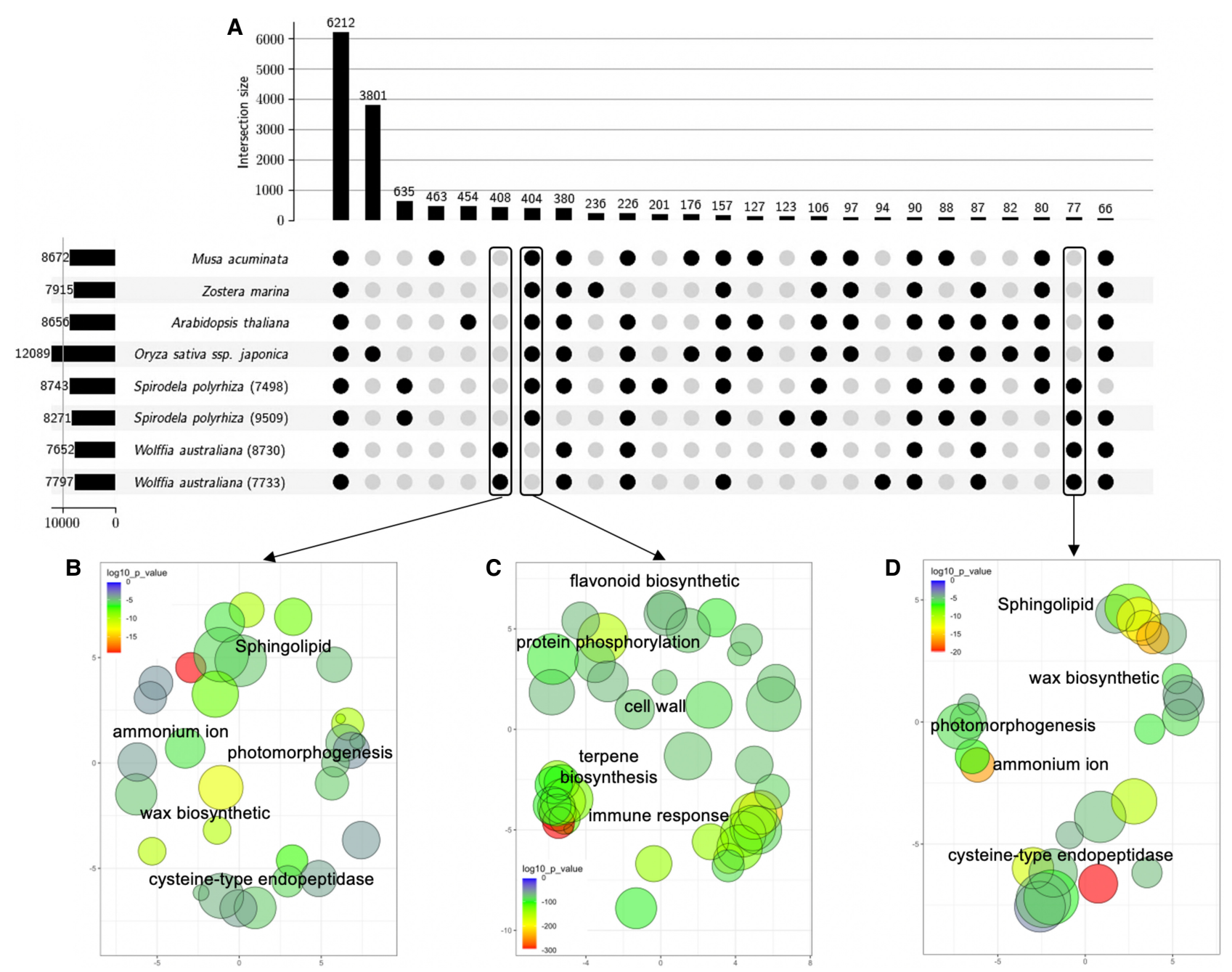

Figure 3. Wolffia orthogroups (OGs) analysis reveals significant Gene Ontology (GO) terms relating to growth and defense. (A) An upset plot showing the orthogroups (OGs) present (black circles) or missing (gray circle) across the four duckweed genomes (sp9509, sp7498, wa7733, and wa8730), model species (Arabidopsis thaliana, Oryza sativa ssp. Japonica), and two non-grass monocots (Musa acuminata, Zostera marina). Boxes indicate Wolffia-specific, Wolffia-missing, and duckweed-specific genes. (B) Significant (FDR $<0.05) \mathrm{GO}$ terms derived from Wolffia-specific OGs $(A)$ were plotted in two-dimensional semantic space by multidimensional scaling (MDS) with the color reflecting significance ( $P$-value) and circle size reflecting GO frequency. (C) GO terms from Wolffia missing OGs. (D) GO terms from duckweed-specific OGs. All GO terms were summarized using semantic similarity (SimRel), and $P$-values from overrepresentation with REVIGO.

provide innate immunity to pathogen-associated molecular patterns or effector triggered resistance responses that are associated with systemic activation of broad spectrum immunity (Jones and Dangl 2006). Members of this family of proteins are known to be the most rapidly evolving genes in plants, and they are likely under strong selection pressure (van Wersch and Li 2019). In the sp9509 genome, we have previously annotated only 58 NLR genes in contrast to the 178 NLRs that are known in Arabidopsis and 387 in Brachypodium (Michael et al. 2017). In the wa7733 and wa8730 genomes, we found only a single canonical NLR gene. Although it has conserved homologs in the Spirodela genomes, it is very divergent from NLR genes of other species. Additionally, two NLR-like genes that contained incomplete NB-ARC domains were identified in the Wolffia genome assemblies, and these genes are highly conserved across duckweeds, Arabidopsis, and rice.

Another gene loss was associated with light signaling and the circadian clock in Wolffia. We reasoned that because Wolffia has apparently optimized for fast growth through rapid multiplication that it may have expanded light signaling and circadian gene families to better fine-tune its physiological response to the environment. However, we observed the opposite with Wolffia, which only had one-third of light signaling and circadian clock genes compared to that in other land plants (30 vs. 90) and half of those found in Spirodela and Zostera (Supplemental Table S11). Of the conserved single-copy BUSCO genes that are also light and circadian-related, Wolffia is missing WITH NO LYSINE (K) KINASE 1 (WNK1) (Murakami-Kojima et al. 2002), TANDEM ZINC KNUCKLE (TZP) (Loudet et al. 2008), FAR1-RELATED FACTOR1 (FRF1) (Ma and Li 2018), and several of the light harvesting complex genes. In addition, Wolffia is missing the core clock component TIMING OF CAB EXPRESSION 1 (TOC1/PRR1), which is also missing in Spirodela and Zostera, yet present in all other non-grass monocots. TOC1/PRR1 specifically binds the promoter of CELL DIVISION CONTROL 6 (CDC6) to regulate the time of DNA replication licensing and growth in Arabidopsis (Fung-Uceda et al. 2018). In contrast, Wolffia had similar numbers of proteins for flowering 
time (FLOWERING LOCUS T [FT] and CONSTANS [CO]) and temperature response (HEAT SHOCK PROTEINS [HSP] and C-repeat/ DRE-Binding Factor [CBF]) (Supplemental Table S11).

\section{Time-of-day expression networks}

$W$. australiana doubles in just over a day (Supplemental Table S1), and because growth is controlled through TOD expression networks, we set out to produce a temporally resolved transcriptome data set for one of the two Wolffia accessions. Wa8730 was grown under standard diurnal conditions of photocycles and constant temperature, which is $12 \mathrm{~h}$ of light $(\mathrm{L})$ at $20^{\circ} \mathrm{C}$ and $12 \mathrm{~h}$ of dark (D) at $20^{\circ} \mathrm{C}$ (referred to as LDHH, light/dark/hot/hot) for $3 \mathrm{wk}$ after transfer. Fronds were then sampled every $4 \mathrm{~h}$ over $2 \mathrm{~d}$ for a total of 13 time points (Supplemental Fig. S10). We found that 83\% $(11,870)$ of the predicted genes were expressed significantly under the LDHH condition (Supplemental Fig. S11; Supplemental Table S12). We estimated the number of genes showing cycling behavior using HAYSTACK $(\mathrm{R}>0.8)$ and found that $13 \%$ (1638) of the expressed genes displayed a TOD expression pattern under the LDHH condition (Supplemental Fig. S11; Supplemental Table S13). We also predicted cycling genes using another popular tool called JTK_CYCLE and found slightly fewer cycling genes (11.5\%) with significant overlap with the HAYSTACK results (Supplemental Fig. S12; Supplemental Table S14); all subsequent analyses were conducted with the HAYSTACK results. Wa8730 genes displayed peak expression, or phase, every hour over the day, sim- ilar to TOD time courses under the LDHH condition in other plants, with more genes peaking at morning- or evening-specific phases (Fig. 4E; Supplemental Fig. S13). A possible contributing factor for the reduced number of TOD-controlled genes in Wolffia under the LDHH condition is the lower percentage of transcription factors (TFs) that cycle (11\%) compared to other species like Arabidopsis and rice (Supplemental Table S17).

The phase of expression of core clock genes is conserved across species (Filichkin et al. 2011). Therefore, we looked at the expression of the core clock-related proteins to both validate the time course and establish if their expression is also conserved in Wolffia. At the core of the clock is a family of single MYB (sMYB) domain transcription factors $L H Y$ and CIRCADIAN CLOCK ASSOCIATED 1 (CCA1), along with a related family called REVEILLE (RVE) (McClung 2019). Although Arabidopsis has 12 sMYB genes similar to CCA1/LHY/RVE, Wolffia has four and only two cycle with a dawn phase; one is the likely CCA1/LHY ortholog and the other is orthologous to RVE7 (Fig. 4A). The other half of the core circadian clock negative feedback loop is the evening expressed TOC1, which is missing in Wolffia. TOC1 is part of a five-member gene family of PRRs, which display "waves" of expression across the day (PRR9, dawn; PRR7, midday; PRR5, dusk; PRR3, evening; TOC1/PRR1, evening) (Michael and McClung 2003). Wolffia only had three PRRs (plus three and four ARR for wa7733 and wa8730, respectively): WaPRR9, WaPRR7/3, and WaPRR5, which are phased to dawn, dusk, and evening, respectively (Fig. 4B). Other core circadian proteins such as GIGANTEA (GI), EARLY
A 5.0

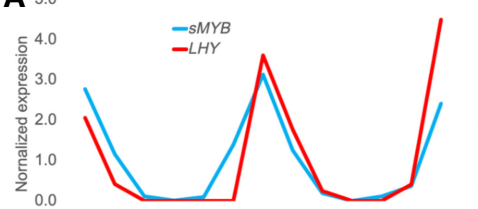

$\mathbf{B}_{3.5}$

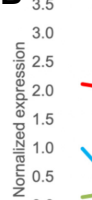

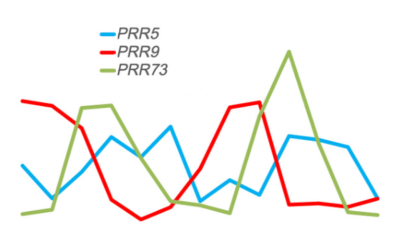

$\mathbf{C}_{6.0}$

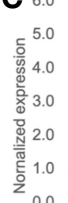

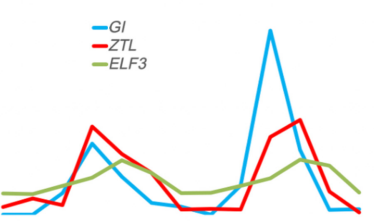

D 2.5

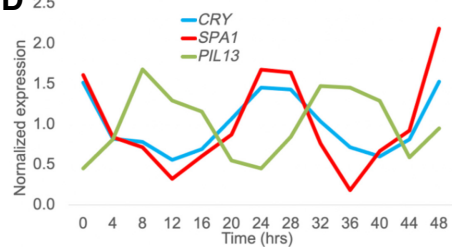

$\mathbf{E}$
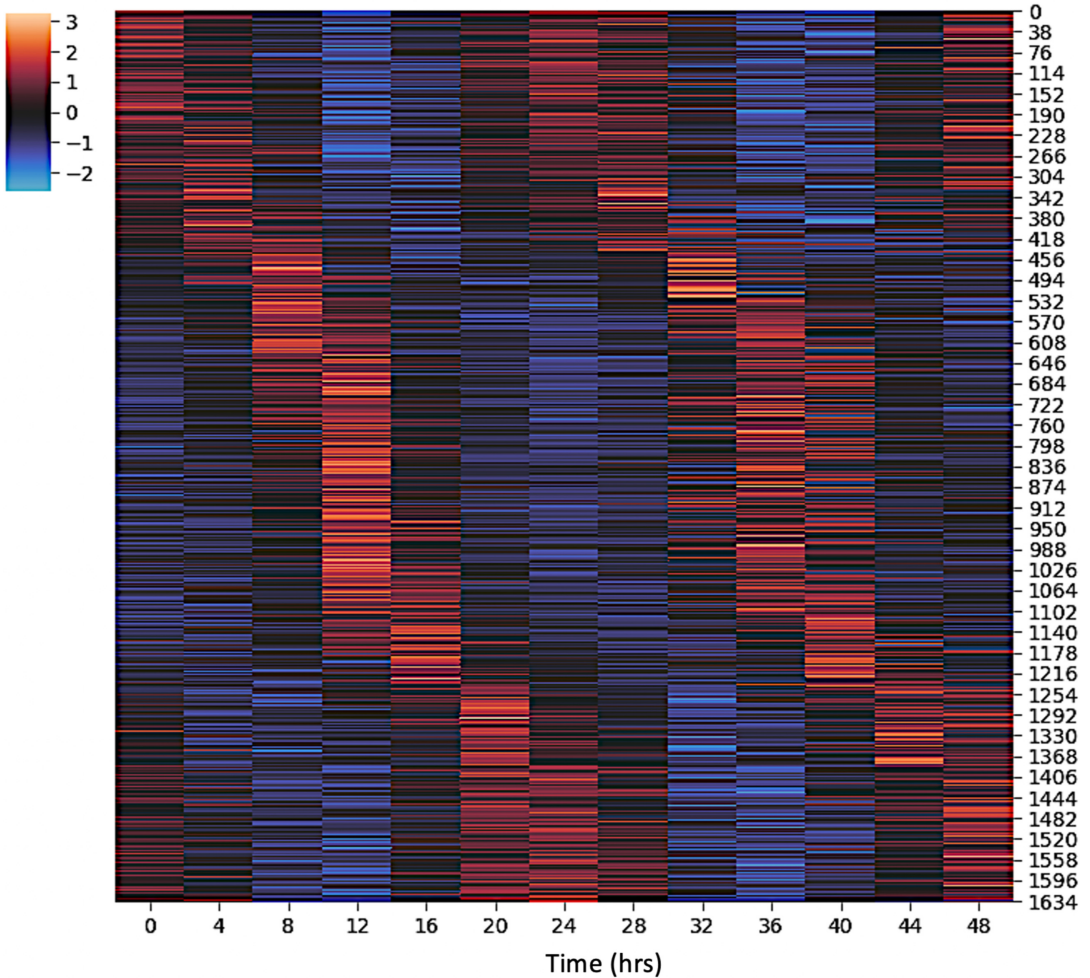

Figure 4. Core circadian and light genes cycle in Wolffia with global expression over the day. (A-D) Circadian clock and light signaling TOD gene expression in wa8730 is similar to that found in other plants. ( $A$ ) $s M Y B$ (blue) and $L H Y$ (red) cycle with dawn specific expression. (B) PRR5 (blue), PRR9 (red), and $P R R 73$ (green) show waves of expression similar to other species. (C) GI (blue), ZTL (red), and ELF3 (green) cycle with evening-specific expression. (D) Light signaling genes CRY (blue), SPA1 (red), and PIL3 (green) cycle over the day. (E) Heatmap of the cycling genes with red indicating high expression and blue indicating low expression. Genes are on the $y$-axis and time is on the $x$-axis.

\section{Genome Research}

www.genome.org 
FLOWERING 3 (ELF3), and FLAVIN-BINDING, KELCH REPEAT, F$B O X$ (FKF1) have evening expression as in other plants (Fig. 4C), as well as circadian-regulated light signaling genes (Fig. 4D). Despite having a reduced set of circadian and light signaling genes (Supplemental Table S11), TOD expression is conserved across the core circadian clock proteins.

As another check of the time course, we looked to see if TOD cis-elements that we have found to be conserved in other plant species are also found in Wolffia (Michael et al. 2008b). We searched promoters (500 bp upstream) of genes predicted to be expressed at the same time of day and found the same cis-elements that we have identified across all other plants tested to date (Zdepski et al. 2008; Michael et al. 2008b; Filichkin et al. 2011). For instance, the Evening Element (EE: AAATATCT), which was identified in early microarray experiments and promoter bashing (Harmer et al. 2000; Michael and McClung 2002), is highly overrepresented in the promoters of evening expressed genes (Fig. 5A,D), whereas the Gbox (CACGTG) and its derivatives are highly overrepresented at dawn (Fig. 5C), and the Protein Box (PBX: GTGGGCCCC) is overrepresented late in the night (Fig. 5E; Supplemental Tables S15, S16). In contrast, the TeloBox (TBX: AAACCCT), which is usually overrepresented in genes expressed around midnight, was not significant in Wolffia (Fig. 5B). The lack of the TBX could partly explain the decreased number of cycling genes or it could mean that Wolffia genes with the TBX do not cycle like they do in Arabidopsis, rice, and poplar (Filichkin et al. 2011). To test these options, we leveraged our empirically proven informatic method to assign cycling and phase information from Arabidopsis to its Wolffia reciprocal best blast (RBB) ortholog (Michael et al. 2008b; Zdepski et al. 2008; Filichkin et al. 2011). We found that the TBX was overrepresented in the promoters of Wolffia orthologs assigned the phase from Arabidopsis, consistent with Wolffia TBXcontaining genes not cycling under the conditions tested (Supplemental Fig. S14). In Arabidopsis, genes associated with protein synthesis and other activities that occur in the middle of the night contain the TBX cis-element, suggesting Wolffia may have lost the TOD coordination for these pathways.

\section{Wolffia cycling genes are focused on core energy acquisition pathways}

Because Wolffia has fewer TOD controlled genes under LDHH, we wanted to know if this was caused by fewer pathways cycling or just a result of Wolffia having fewer genes per family (orthogroups). If the later were true, then we would expect Wolffia to have a similar number of cycling OGs compared to other plants that have expanded gene families. Therefore, we compared the Wolffia cycling genes against two high-quality time courses from Arabidopsis and rice generated under the same LDHH conditions (Michael et al. 2008b; Filichkin et al. 2011). Of the OGs that had at least one gene cycling in Arabidopsis, rice, and Wolffia, we found that 49\% (4293/8724), 74\% (6025/8063), and 18\% (1442/ 7844) were cycling, respectively, which are similar numbers to overall cycling for each species and consistent with larger gene families not playing a significant role in percent cycling in any of the species (Fig. 6B; Supplemental Fig. S15). Eighty-one percent of cycling Wolffia OGs were shared with Arabidopsis and rice, suggesting the cycling pathways in Wolffia are a subset of those found in other plants. In addition, the OGs that did cycle in Wolffia were more likely to share the same mean phase of expression with Arabidopsis and rice, consistent with the conservation of TOD expression that has been found in other studies (Filichkin et al. 2011; de los Reyes et al. 2017; Supplemental Fig. S16).

We next looked to see which Wolffia pathways were cycling, and by proxy through comparison with data from Arabidopsis and rice for those pathways that are not cycling in Wolffia, by looking at the GO terms that were significantly overrepresented at specific times over the day. Wolffia had 92 significant GO terms that are TOD specific, whereas Arabidopsis and rice had 238 and 253, respectively (Fig. 6A; Supplemental Fig. S17; Supplemental Tables
A

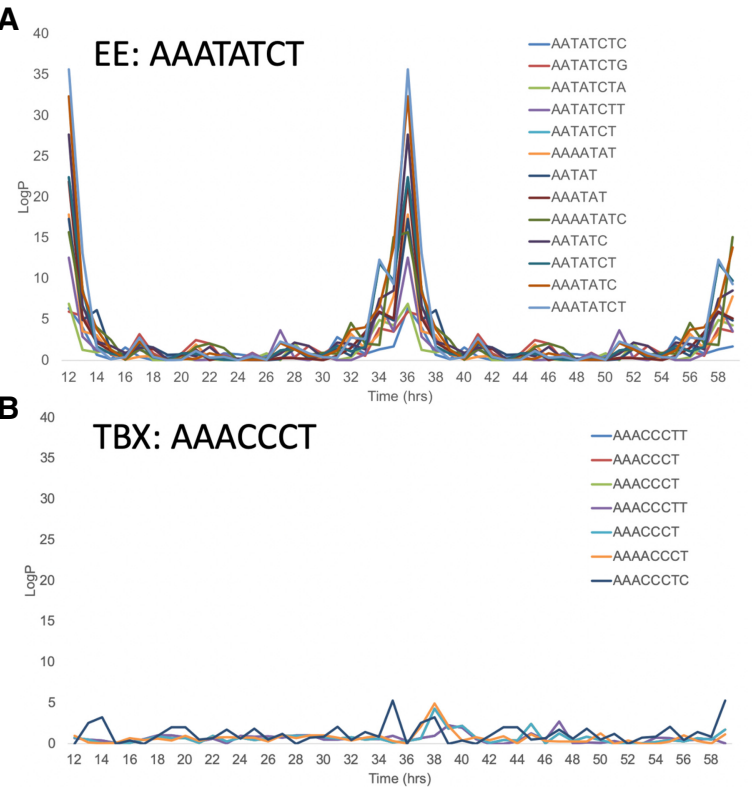

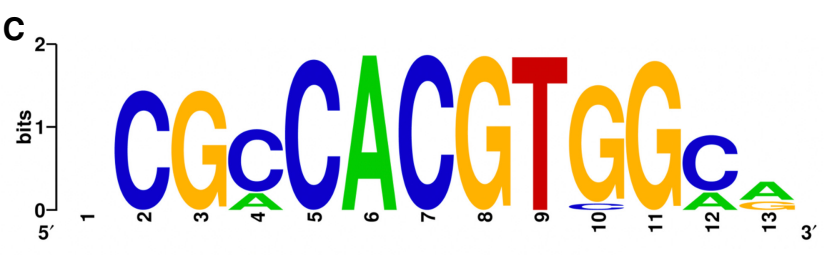
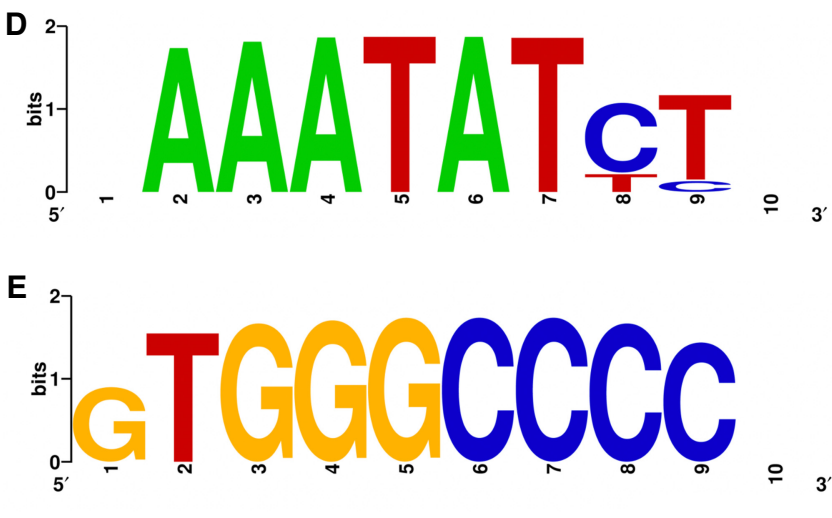

Figure 5. Wolffia has conserved TOD cis-elements but lacks others. (A) The Evening Element (EE:AAATATCT) is overrepresented in genes with eveningspecific expression. (B) The Telobox (TBX:AAACCCT) is not significantly overrepresented in Wolffia promoters of cycling genes. (C) Sequence logo of the significantly overrepresented Gbox (CACGTG) in Wolffia. $(D)$ Sequence logo of the EE overrepresented cis-elements. $(E$ ) Sequence logo of the Protein box (PBX: TGGGCCC) overrepresented cis-elements. 
A GO term TOD significance

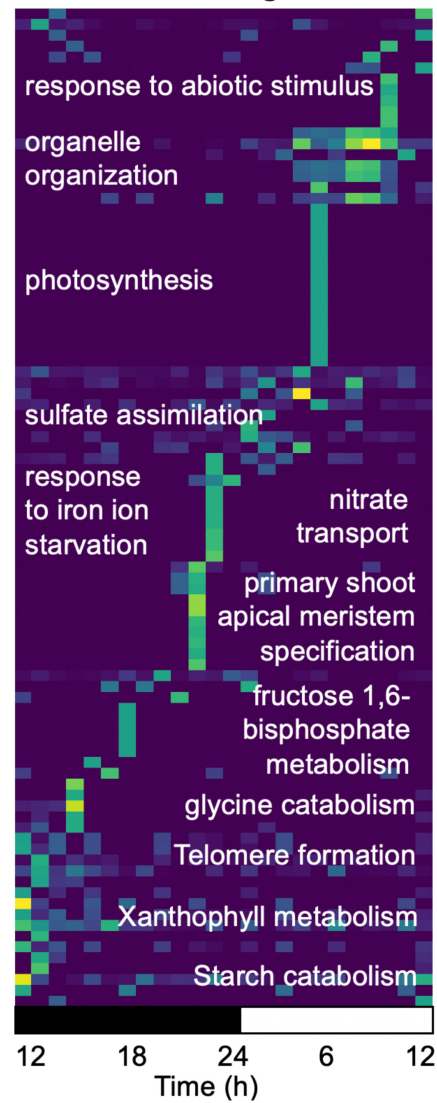

B
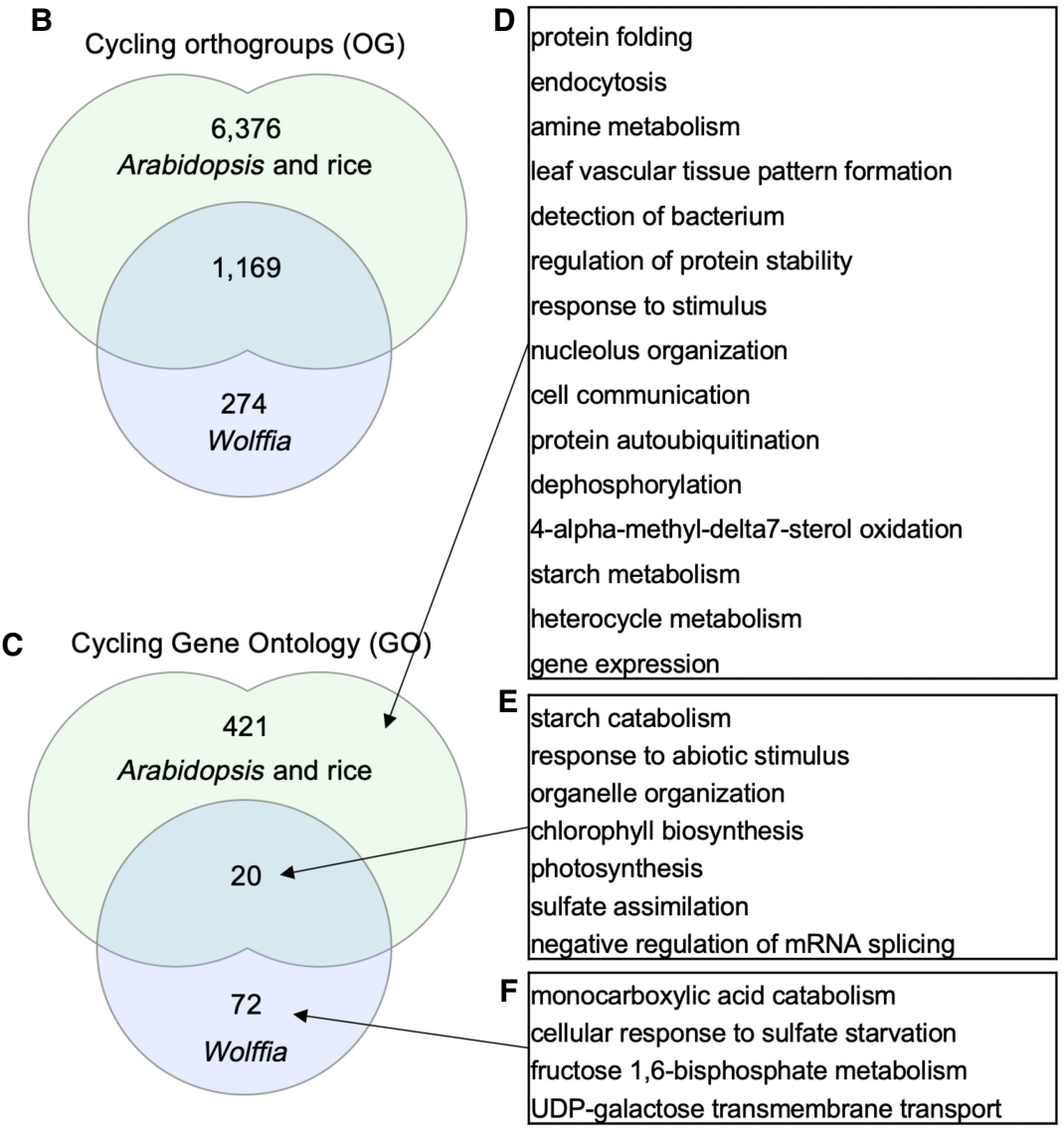

E

starch catabolism response to abiotic stimulus organelle organization chlorophyll biosynthesis photosynthesis sulfate assimilation negative regulation of $\mathrm{mRNA}$ splicing

Fonocarboxylic acid catabolism cellular response to sulfate starvation fructose 1,6-bisphosphate metabolism UDP-galactose transmembrane transport

Figure 6. Wolffia cycling genes are focused on energy acquisition. ( $A$ ) GO term overrepresentation by TOD for Wolffia with GO term summaries for 6-h bins. (B) Venn diagram of cycling orthogroups (OGs) with at least one gene from Wolffia, Arabidopsis, and rice. (C) Venn diagram of cycling GO terms from Wolffia, Arabidopsis, and rice. (D) GO term summary for the 421 Arabidopsis and rice cycling GO terms. (E) GO term summary for the 20 cycling GO terms shared across all three species. $(F)$ GO term summary for the 72 Wolffia-specific cycling GO terms. All GO terms were summarized using semantic similarity (SimRel), and $P$-values from overrepresentation with REVIGO.

S18-S20). The 20 significant GO terms shared across all three species were summarized into sulfate assimilation, response to abiotic stimulus, ribosomal small subunit biogenesis, photosynthesis, photosystem I, starch catabolism, and chlorophyll biosynthesis (Fig. 6C,E). The remaining 72 Wolffia-specific TOD GO terms also are focused on carbon metabolism and summarized into monocarboxylic acid catabolism, cellular response to sulfate starvation, fructose 1,6-bisphosphate metabolism, and UDP-galactose transmembrane transport (Fig. 6F). Both the shared and speciesspecific significant TOD GO terms are focused on energy acquisition in Wolffia. In contrast, the 421 significant GO TOD terms in Arabidopsis or rice are summarized into 15 biological processes consistent with plants that have more complex structure (Fig. 6D) (Michael et al. 2008b; Filichkin et al. 2011). In addition, several of the summarized GO terms that are missing in Wolffia, but found in Arabidopsis and rice, are related to protein biosynthesis pathways, which is consistent with the loss of the TBX cis-element in Wolffia (Fig. 5B; Supplemental Fig. S14). These results show that Wolffia has retained a core set of cycling genes focused on energy acquisition and utilization, but other pathways common in model plants have been released from TOD control. Because the primary role of the circadian clock is to gate processes to coincide with specific times of day (Michael et al. 2003), and the circadian clock is dispensable in plants, the reduced TOD control in Wolffia could reflect its less orchestrated continuous growth pattern.

\section{Discussion}

Here, we present two draft genomes for different accessions of $W$. australiana, which has a relatively small genome and contains a minimal set of about 15,000 genes. A key finding of our work is that Wolffia has a reduced number of TOD-regulated genes (13\%) compared to other plants under the same assay conditions, and the genes that remain regulated are specific to photosynthesis and carbon metabolism. Because Wolffia is small ( $\sim 1 \mathrm{~mm}$ in size), fast growing (DT 1 d), has a minimal set of core plant genes, and grows in direct contact with the media, it offers advantages analogous to the yeast system, which opens up new research opportunities in plants.

$W$. australiana has the smallest genome across the 11 species in the genus tested, which have an average size of $1136 \mathrm{Mb}$, and is half the size of the next smallest species of $W$. brasiliensis at $776 \mathrm{Mb}$ (Wang et al. 2011). Despite the range in genome sizes, all Wolffia species have a reduced body plan of a frond with just several thousand cells and no roots (Fig. 1). They also have a similar fast growth rate of around a day (Supplemental Table S1) with no obvious

\section{Genome Research}

www.genome.org 
relationship to genome size (Sree et al. 2015b). Wolffia contains the most derived species of all the duckweeds (Fig. 1C), with body plan reductions compared to the most basal Spirodela genus. The results that several key genes associated with root and lightspecific development were disrupted by LTR-RT in $W$. australiana versus Spirodela suggests that at some level the changes in morphology are the result of TE activity. Like Spirodela, which also has a small genome, the LTR-RT solo::intact ratio is high in $W$. australiana consistent with LTR-RT being purged through illegitimate recombination (Devos et al. 2002). Therefore, it is probable that $W$. australiana has a relatively small genome compared to other Wolffia species owing to the active purging of LTR-RT that in the past has helped to shape its unique gene repertoire and body plan.

Wolffia, and the Lemnaceae family in general, represent extreme examples of plant morphology minimization, and these adaptations are reflected in their reduced yet representative gene sets. Multiple independently assembled genomes of both Spirodela and Wolffia share a common set of missing BUSCO genes (Supplemental Fig. S7), and many of these genes in Wolffia represent genes associated with its morphological innovations (Fig. 3; Supplemental Table S6). Low BUSCO scores have also been noted in other plants with morphological innovations like the parasitic dodder plant (Cuscuta) and carnivorous bladderwort (Utricularia) that both also lack roots and leaf structures like Wolffia (Ibarra-Laclette et al. 2013; Sun et al. 2018; Vogel et al. 2018). Cuscuta australis shares many of the same gene losses as Wolffia such as WOX5 (root apical stem cell maintenance), LOP1 (leaf patterning and root development), and the entire CASP family (casparian strip) (Sun et al. 2018). Wolffia is also missing several families of the small signaling peptide CLAVATA3/ESR-RELATED (CLE) that regulate various aspects of cell fate and meristem size (Jun et al. 2010). Loss of CLE3 peptide known to play key roles in orchestrating meristem size in plants is correlated with the unconventional mode of organogenesis in duckweed in which the stipe tissue functions as the site where new meristems are continuously initiated and develop sequentially (Fig. 1C; Lemon and Posluszny 2000). Despite the extensive gene loss, Wolffia still maintains a core set of gene families (OGs) common to flowering plants that have minimal expansions (Supplemental Table S7; Supplemental Fig. S9).

Wolffia has lost a host of genes associated with the intersection between light signaling, phytohormones, circadian clock, growth, immunity, and development that may provide insight into its floating ball-shaped morphology. It is critical for a multiorgan plant to position itself relative to the Earth (gravitropism) and the sun (phototropism) for proper development, as well as the means to communicate between the different parts of the organism through systemic signaling (Vandenbrink et al. 2014). Wolffia has lost many genes of the NPH3/RPT2-Like family $(N R L)$, which are required for several auxin-mediated growth processes, including phototropism (root and shoot), petiole positioning, leaf expansion, chloroplast accumulation, stomatal opening, and circadian control of PSII photosynthetic efficiency (Christie et al. 2018). NPH3 is the founding member of the NRL family with a close paralog DOT3, mutants of which fail to show phototropism and have aberrant venation patterning in Arabidopsis, respectively (Liscum and Briggs 1995; Motchoulski and Liscum 1999; Petricka et al. 2008). Compared to Arabidopsis, Spirodela has only one NPH3/DOT3, which is lost in Wolffia through an LTR-RT disruption (Fig. 2D). In addition, Wolffia is missing the family of $L A Z Y$ proteins that act as central integrators of gravity sensing with the formation of auxin gradients to control plant architecture (Yoshihara and Spalding 2017). The loss of these two key phototropic and gravitropic pathways provide clues as to how Wolffia has streamlined its body plan.

Lastly, the attrition of the highly conserved NLR family that are known to contain many $\mathrm{R}$ genes in plants required to mediate defense signaling and systemic resistance indicates that these genes are largely dispensable for a fast-growing and structurally simple plant. Elucidating how the stipe tissue continually functions to generate new meristematic centers that are orchestrated to produce new daughters and the mechanism that provides Wolffia with robust defense to pathogens in an R-gene independent manner will reveal much new plant biology. For the latter, the emphasis of the sphingolipid-related pathways observed in our work here may suggest their importance for defense signaling in Wolffia while there is also some evidence for an amplification of the antimicrobial peptide pathways in Spirodela (An et al. 2019). These suggestions remain to be examined in greater detail and tested in the future.

One of the best described plant growth processes at the molecular level is that of hypocotyl elongation in Arabidopsis (Creux and Harmer 2019). The circadian clock restricts or "gates" the growth during the night hours (dark) through the core clock protein TOC1 binding to the PHYTOCHROME INTERACTING FACTORS (PIF) transcription factors and interaction with one of the core feedback loops mediated by the Evening Complex (EC: ELF3/ELF4/LUX) (Seluzicki et al. 2017). Wolffia, Spirodela, and Zostera all are missing TOC1 while having several other PRRs, suggesting that they may replace TOC1 function in aquatic non-grass monocots. In addition, Wolffia is missing the orthologs for PIF3/4 while having the other factors of the EC. Also, light-regulated growth is controlled at some level by two distinct types of nuclear photobodies, one of which is defined by TZP (Huang et al. 2016). Loss of TZP in Arabidopsis results in smaller plants, whereas overexpression results in large plants that do not stop growing, consistent with it being a central integrator of light (PHYA) signaling into plant growth (Loudet et al. 2008; Huang et al. 2016; Zhang et al. 2018). Overall, the loss of growth gating pathways in Wolffia is consistent with the significant decrease in circadian, light, and flowering time genes (Supplemental Table S11), which is in contrast to some Crassulacean acid metabolism (CAM) and crop plants that display expansion of circadian genes (Lou et al. 2012; Wai et al. 2019). These results suggest that the genome innovations responsible for the change in body plan in Wolffia may be closely linked to the loss of specific light-gated growth.

The finding that Wolffia has fewer cycling genes under LDHH conditions presents an unexpected paradox when compared with other minimalist organisms that have been studied. Ostreococcus tauri is a single-cell alga that is the smallest eukaryote (picoeukaryote) with a 13-Mb genome but a functional circadian clock made up of the core negative feedback loop of CCA1-PPR1 and almost all of the genes cycling in a TOD fashion (Monnier et al. 2010). Similarly, the model microalga Chlamydomonas reinhardtii has a core circadian clock and $80 \%$ of its genes cycle in a TOD fashion (Zones et al. 2015). In contrast, multicellular, multiorgan plants tested under the LDHH condition have a higher number of cycling genes, such as Arabidopsis (45\%), rice (41\%), poplar (30\%), douglas-fir (29\%), and Brachypodium (27\%) (Michael et al. 2008b; Filichkin et al. 2011; Cronn et al. 2017; MacKinnon et al. 2020). Therefore, it would seem that a simplified (i.e., fewer celled) plant like Wolffia would have almost all of its transcriptome TOD regulated and multiorgan plants would have some processes that would not require TOD expression. The fact that only the core photosynthetic and carbon pathways remain under TOD control 
under LDHH suggests that most processes in Wolffia are uncoupled from the environment.

However, it has been noted that a reduced number of genes ( $5.2 \%)$ cycle in Norway spruce seedings (Picea abies), which could be species-specific or reflect the age of the plants sampled, because other gymnosperms, under normal spring-summer conditions, have a similar number of cycling genes as other model plants (Cronn et al. 2017; Ferrari et al. 2019). In gymnosperms the hypothesis is that in overwintering needles that are not growing, only core photosynthesis is required for coupling to the environment. In both cases, perhaps the common driving force to minimize TOD control of genes is to economize on energy expenditure to survive harsh winters for gymnosperms and rapid, continuous growth for Wolffia. This would be consistent with the retention of TOD control for genes involved in energy acquisition and storage for both cases. In a similar way, it is formally possible that Wolffia just has fewer genes cycling under the condition tested (LDHH), although it has been shown that there is a high level of overlap of cycling genes across environmental conditions (Michael et al. 2008b; Filichkin et al. 2011).

Because Wolffia is in direct contact with the environment (water) where temperature and nutrients are most likely in a relatively constant state over the course of the day, it is possible that Wolffia has uncoupled these processes from TOD expression required in an environment on land. Although most conserved TOD cis-elements are found (Fig. 5; Michael et al. 2008b), the loss of TOD overrepresentation of the TBX in Wolffia, but yet the identification of it through cycling-orthologs with Arabidopsis (Supplemental Fig. S14), suggest that TOD regulation of the highly conserved TBX-controlled pathways (such as protein synthesis) have been lost in Wolffia (Filichkin et al. 2011). The absence of TBX regulation could reflect a general loss of key regulatory switches (fewer TFs) associated with the circadian clock and light signaling (Supplemental Tables S11, S16, S17). Because many of the gene losses in Wolffia link development with light signaling, it is possible that the evolutionary path to a highly reduced plant with simple architecture and continuous growth also resulted in the loss of light-specific gated growth.

Wolffia is like the yeast of flowering plants with a core set of angiosperm genes, small size, rapid unrestricted growth, and growing in direct contact with its environment. Before Arabidopsis, duckweed was widely used as a model plant in plant biology (Lam et al. 2014). In fact, duckweeds were central in elucidating photoperiodic flowering (Lemna perpusilla) and auxin biosynthetic pathways by radioisotope labeling (Lemna gibba) (Hillman 1976; Rapparini et al. 1999). Wolffia still has the genes for flowering and could be developed as a genetic system that is distinct from that of Arabidopsis, where crosses may be made by mixing flowering strains and collecting the seeds that sink to the bottom of the growth medium. The limited number of cells and cell types in Wolffia could provide a simplified model to dissect cell-specific regulation and how plant cells directly respond to specific chemicals at the organismal level.

\section{Methods}

\section{Growth}

W. australiana (Benth.) Hartog \& Plas clones 7211 (Australia, Victoria), 7540 (New Zealand), 7733 (Australia, South Australia), and 8730 (Australia, New South Wales) were maintained at the Rutgers Duckweed Stock Cooperative (http://www.ruduckweed .org/) or at the stock collection at the University of Jena, Germany. The specific growth conditions for growth assays, imaging, and the TOD time course are detailed in the Supplemental Materials and in Appenroth et al. (1996). Explanation of relative growth rate (RGR), doubling time (DT) and relative (weekly) yield (RY) have been previously described (Ziegler et al. 2015). The stack of 311 Wolffia microimages was obtained using digital microscope Keyence VHX-5000 with 600× lens magnification (Keyence Deutschland $\mathrm{GmbH}$ ) and ImageJ software (Schindelin et al. 2012).

\section{Genome sequencing and assembly}

HMW genomic DNA was isolated from young teff leaf tissue for both PacBio and Illumina sequencing. A modified nuclei preparation was used to extract HMW gDNA, and residual contaminants were removed using phenol-chloroform purification (Lutz et al. 2011). Genome size was estimated by $k$-mer frequency with Jellyfish and GenomeScope (Vurture et al. 2017), as well as by flow cytometry as previously described (Hoang et al. 2019). PacBio data was error corrected and assembled using Canu (v1.5) (Koren et al. 2017), and assembly graphs were visualized after each iteration of Canu in Bandage (Wick et al. 2015). A consensus was first generated using the PacBio reads and three rounds of racon (v1.3.1) (Vaser et al. 2017). The raw PacBio contigs were polished to remove residual errors with Pilon (v1.22) (Walker et al. 2014) using Illumina paired-end sequence. Illumina reads were quality-trimmed using Trimmomatic followed by aligning to the assembly with Bowtie 2 (v2.3.0) (Langmead and Salzberg 2012) under default parameters. To test completeness, the Waksman Student Scholars Program (WSSP) (https://www.ncbi.nlm.nih .gov/nuccore/?term $=$ Waksman \pm Student \pm Scholars \pm program \pm wolf fia \pm australiana) cDNA sequences were mapped to the wa8730 and wa7733 genome assemblies using minimap2 (v2.17-r941) (Li 2018). Bionano optical maps were prepared as previously described (Kawakatsu et al. 2016) with minor modifications (Supplemental Materials). Assemblies (wa8730, wa7733, sp9509, sp7498) were benchmarked using the BUSCO (v3) liliopsida odb10 database (Simão et al. 2015). Identification of high copy number repeats such as rDNA and centromere arrays in the two Wolffia accessions were performed as previously described (Hoang et al. 2018).

\section{Genome annotation}

Custom repeat libraries were constructed for each species following the MAKER-P basic protocol (Campbell et al. 2014). RepeatModeler (v1.0.8) was run against the genome assembly to produce an initial de novo library (Smit and Hubley 2008-2015). Sequences with BLASTX hits $\left(E\right.$-value $\left.1 \times 10^{-10}\right)$ to a UniProt database of plant protein-coding genes were removed along with 50-bp flanking sequences. The resulting custom library was used with RepeatMasker (v4.0.7) with default settings (Smit et al. 2015). Protein-coding genes were annotated for all four duckweed genomes with the MAKER 3.01 .02 pipeline (Holt and Yandell 2011). For protein-coding gene predictions, a comprehensive transcriptome assembly for each species was developed using PASA (Haas et al. 2003). Illumina RNA-seq reads for both sp9509 and wa8730 were trimmed with skewer (v0.2.2) (Jiang et al. 2014), aligned to the genome assembly with HISAT2 (v2.1.0) (Kim et al. 2015), and assembled with Trinity (v2.6.6) (Grabherr et al. 2011; Haas et al. 2013). EST sequences from sp7498 (Wang et al. 2014) were downloaded from the NCBI Sequence Read Archive (SRA; https://www.ncbi.nlm.nih.gov/sra) (SRR497624) and assembled with Newbler (v3.0) (Margulies et al. 2005). All available assembled duckweed transcripts were passed to MAKER as evidence. Protein

\section{Genome Research}

www.genome.org 
homology evidence consisted of all UniProtKB/Swiss-Prot (Schneider et al. 2009; UniProt Consortium 2019) plant proteins and the following proteomes: Arabidopsis thaliana, Elaeis guineensis, Musa acuminata, Oryza sativa, Spirodela polyrhiza, and Zostera marina (Goodstein et al. 2012; Singh et al. 2013). Three different approaches were used for ab initio gene prediction. RNA-seq alignments along with the soft-masked assembly were passed to BRAKER 2.1.0 to train species-specific parameters for AUGUSTUS (v3.3.3) and produce the first set of coding gene predictions (Stanke et al. 2006, 2008; Hoff et al. 2016, 2019). A second set of predictions was produced by generating a whole-genome multiple sequence alignment of all duckweed species with Cactus (Paten et al. 2011) and running AUGUSTUS in CGP mode (König et al. 2016). The final set of predictions was produced by running AUGUSTUS within the MAKER pipeline, using the BRAKER-generated species parameters along with protein and assembled RNAseq alignments passed as evidence. MAKER was run and allowed to select the gene models most concordant with the evidence among these three prediction data sets. MAKER-P (Campbell et al. 2014) standard gene builds were generated by running InterProScan (v5.30-69) (Jones et al. 2014) and retaining only those predictions with a Pfam domain or having evidence support (AED score $<1.0)$. Orthogroups and orthologs were identified across 29 proteomes from the Plaza 4.0 Monocots database (Van Bel et al. 2018) and the duckweed MAKER-P standard build proteomes with alternate transcripts removed. OrthoFinder (v2.2.7) (Emms and Kelly 2015, 2019) was run against all-versus-all proteome alignments computed with DIAMOND (Buchfink et al. 2015) using the standard workflow. To create a consistent set of GO term classifications for every protein in each species in the data set, the 28 Plaza monocot (v4) proteomes and the four duckweed proteomes were processed by eggNOG-mapper (Huerta-Cepas et al. 2017). The significant GO terms (FDR <0.05) were summarized and visualized using REVIGO (Supek et al. 2011). The nucleotide-binding leucine-rich repeat proteins (NLRs) were predicted using NLR-Annotator (Steuernagel et al. 2020) that scans genomic sequences for MEME-based sequence motifs (Bailey et al. 2009). In addition, the proteomes were queried for the presence of the NB-ARC domain (PF00931) (Sarris et al. 2016). Genomic and proteomic NLR predictions were combined to create a nonredundant list of putative NLRs, each putative NLR with an available gene model was then run through interpro-scan (Jones et al. 2014) for further domain prediction. The set of NLRs from either accession and species were then aligned by MAFFT (Katoh and Standley 2016) to identify orthologs.

\section{Time-of-day time course analysis}

TOD time course data was analyzed with similar methods that have been described with some modifications described below (Michael et al. 2008b; Filichkin et al. 2011; Wai et al. 2019; MacKinnon et al. 2020). HISAT2 (Kim et al. 2015) was used to align RNA-seq reads to the wa8730 assembly. The resultant alignments were processed by Cuffquant and CuffNorm (Trapnell et al. 2012) to generate normalized expression counts for each gene for each time point. Genes with mean expression across the 13 time points below 1 FPKM were filtered before being processed by HAYSTACK (Michael et al. 2008b). As a check, cycling genes were also predicted cycling genes using JTK_CYCLE (Hughes et al. 2010). Once cycling genes in wa8730 were identified, we were able to find putative cis-acting elements associated with TOD expression. Promoters, defined as $500 \mathrm{bp}$ upstream of genes, were extracted for each gene in wa8730 and processed by ELEMENT (Mockler et al. 2007; Michael et al. 2008a,b). Our threshold for identifying a $k$-mer as being associated with cycling was an FDR $<0.05$ in at least one of the comparisons.

\section{Data access}

The raw PacBio data, Illumina resequencing, and RNA-seq reads generated in this study were deposited to the NCBI BioProject database (https://www.ncbi.nlm.nih.gov/bioproject/) under accession number PRJNA615235 (for individual SRA accession numbers, see Supplemental Table S21). The genome assemblies wa8730 and wa7733 are available from CoGe (https ://genomevolution.org/) under Genome ID 56605 and 56606, respectively.

\section{Competing interest statement}

The authors declare no competing interests.

\section{Acknowledgments}

This material is based on work supported by the U.S. Department of Energy, Office of Science, Office of Biological and Environmental Research program under Award Number DESC0018244. Duckweed research at the Lam Laboratory is also supported in part by a grant from Hatch project (\#12116) from the New Jersey Agricultural Experiment Station at Rutgers University. R.A.M. and J.R.E. are investigators of the Howard Hughes Medical Institute.

Author contributions: T.P.M. and E.L. conceived the study, oversaw the experiments, and wrote the manuscript; D.B. and T.C.M. assembled the genome; F.J., J.P.S., and J.R.E. generated BioNano optical maps and scaffolded the genome; K.S.S. and K.J.A. conducted growth experiments; K.J.A. and J.F. performed genome size estimations with flow cytometry; S.O. and L.B. performed sectioning, microscopy, and developed images and timelapse video of Wolffia; P.C. and S.G. carried out tissue sampling and RNA isolation for the circadian transcriptome study; E.E. and R.A.M. predicted protein-coding genes and protein family (orthogroup) analysis; E.L.B. and K.V.K. performed NB-ARC gene curation and analysis; and T.P.M. and N.H. conducted orthogroups, Gene Ontology terms, and TOD analyses. All authors have read and approved the manuscript.

\section{References}

An D, Zhou Y, Li C, Xiao Q, Wang T, Zhang Y, Wu Y, Li Y, Chao DY, Messing J, et al. 2019. Plant evolution and environmental adaptation unveiled by long-read whole-genome sequencing of Spirodela. Proc Natl Acad Sci 116: 18893-18899. doi:10.1073/pnas.1910401116

Appenroth KJ, Teller S, Horn M. 1996. Photophysiology of turion formation and germination in Spirodela polyrhiza. Biol Plant 38: 95.

Bailey TL, Boden M, Buske FA, Frith M, Grant CE, Clementi L, Ren J, Li WW, Noble WS. 2009. MEME SUITE: tools for motif discovery and searching. Nucleic Acids Res 37: W202-W208. doi:10.1093/nar/gkp335

Bernard FA, Bernard JM, Denny P. 1990. Flower structure, anatomy and life history of Wolffia australiana (Benth.) den Hartog \& van der Plas. Bull Torrey Bot Club 117: 18-26. doi:10.2307/2997125

Bog M, Lautenschlager U, Landrock MF, Landolt E, Fuchs J, Sowjanya Sree K, Oberprieler C, Appenroth KJ. 2015. Genetic characterization and barcoding of taxa in the genera Landoltia and Spirodela (Lemnaceae) by three plastidic markers and amplified fragment length polymorphism (AFLP). Hydrobiologia 749: 169-182. doi:10.1007/s10750-014-2163-3

Borisjuk N, Chu P, Gutierrez R, Zhang H, Acosta K, Friesen N, Sree KS, Garcia C, Appenroth KJ, Lam E. 2015. Assessment, validation and deployment strategy of a two-barcode protocol for facile genotyping of duckweed species. Plant Biol 17: 42-49. doi:10.1111/plb.12229

Buchfink B, Xie C, Huson DH. 2015. Fast and sensitive protein alignment using DIAMOND. Nat Methods 12: 59-60. doi:10.1038/nmeth.3176 
Campbell MS, Law M, Holt C, Stein JC, Moghe GD, Hufnagel DE, Lei J, Achawanantakun R, Jiao D, Lawrence CJ, et al. 2014. MAKER-P: a tool kit for the rapid creation, management, and quality control of plant genome annotations. Plant Physiol 164: 513-524. doi:10.1104/pp.113 .230144

Christie JM, Suetsugu N, Sullivan S, Wada M. 2018. Shining light on the function of NPH3/RPT2-like proteins in phototropin signaling. Plant Physiol 176: 1015-1024. doi:10.1104/pp.17.00835

Creux N, Harmer S. 2019. Circadian rhythms in plants. Cold Spring Harb Perspect Biol 11: a034611. doi:10.1101/cshperspect.a034611

Cronn R, Dolan PC, Jogdeo S, Wegrzyn JL, Neale DB, St Clair JB, Denver DR. 2017. Transcription through the eye of a needle: daily and annual cyclic gene expression variation in Douglas-fir needles. BMC Genomics 18: 558. doi:10.1186/s12864-017-3916-y

de los Reyes P, Romero-Campero FJ, Teresa Ruiz M, Romero JM, Valverde F. 2017. Evolution of daily gene co-expression patterns from algae to plants. Front Plant Sci 8: 1217. doi:10.3389/fpls.2017.01217

Devos KM, Brown JKM, Bennetzen JL. 2002. Genome size reduction through illegitimate recombination counteracts genome expansion in Arabidopsis. Genome Res 12: 1075-1079. doi:10.1101/gr.132102

Dodd AN, Salathia N, Hall A, Kévei E, Tóth R, Nagy F, Hibberd JM, Millar AJ, Webb AAR. 2005. Plant circadian clocks increase photosynthesis, growth, survival, and competitive advantage. Science 309: 630-633. doi:10.1126/science.1115581

Emms DM, Kelly S. 2015. OrthoFinder: solving fundamental biases in whole genome comparisons dramatically improves orthogroup inference accuracy. Genome Biol 16: 157. doi:10.1186/s13059-015-0721-2

Emms DM, Kelly S. 2019. OrthoFinder: phylogenetic orthology inference for comparative genomics. Genome Biol 20: 238. doi:10.1186/s13059019-1832-y

Ferrari C, Proost S, Janowski M, Becker J, Nikoloski Z, Bhattacharya D, Price D, Tohge T, Bar-Even A, Fernie A, et al. 2019. Kingdom-wide comparison reveals the evolution of diurnal gene expression in Archaeplastida. Nat Commun 10: 737. doi:10.1038/s41467-019-08703-2

Filichkin SA, Breton G, Priest HD, Dharmawardhana P, Jaiswal P, Fox SE, Michael TP, Chory J, Kay SA, Mockler TC. 2011. Global profiling of rice and poplar transcriptomes highlights key conserved circadian-controlled pathways and cis-regulatory modules. PLoS One 6: e16907. doi:10 .1371/journal.pone.0016907

Fung-Uceda J, Lee K, Seo PJ, Polyn S, De Veylder L, Mas P. 2018. The circadian clock sets the time of DNA replication licensing to regulate growth in Arabidopsis. Dev Cell 45: 101-113.e4. doi:10.1016/j.devcel.2018.02 .022

Goodstein DM, Shu S, Howson R, Neupane R, Hayes RD, Fazo J, Mitros T, Dirks W, Hellsten U, Putnam N, et al. 2012. Phytozome: a comparative platform for green plant genomics. Nucleic Acids Res 40: D1178-D1186. doi:10.1093/nar/gkr944

Grabherr MG, Haas BJ, Yassour M, Levin JZ, Thompson DA, Amit I, Adiconis X, Fan L, Raychowdhury R, Zeng Q, et al. 2011. Full-length transcriptome assembly from RNA-Seq data without a reference genome. Nat Biotechnol 29: 644-652. doi:10.1038/nbt.1883

Green RM, Tingay S, Wang ZY, Tobin EM. 2002. Circadian rhythms confer a higher level of fitness to Arabidopsis plants. Plant Physiol 129: 576-584. doi:10.1104/pp.004374

Haas BJ, Delcher AL, Mount SM, Wortman JR, Smith RK, Hannick LI, Maiti $\mathrm{R}$, Ronning $\mathrm{CM}$, Rusch $\mathrm{DB}$, Town $\mathrm{CD}$, et al. 2003. Improving the Arabidopsis genome annotation using maximal transcript alignment assemblies. Nucleic Acids Res 31: 5654-5666. doi:10.1093/nar/gkg770

Haas BJ, Papanicolaou A, Yassour M, Grabherr M, Blood PD, Bowden J, Couger MB, Eccles D, Li B, Lieber M, et al. 2013. De novo transcript sequence reconstruction from RNA-seq using the trinity platform for reference generation and analysis. Nat Protoc 8: 1494-1512. doi:10.1038/ nprot.2013.084

Harmer SL, Hogenesch JB, Straume M, Chang HS, Han B, Zhu T, Wang X, Kreps JA, Kay SA. 2000. Orchestrated transcription of key pathways in Arabidopsis by the circadian clock. Science 290: 2110-2113. doi:10 $.1126 /$ science.290.5499.2110

Hillman WS. 1976. Calibrating duckweeds: light, clocks, metabolism, flowering. Science 193: 453-458. doi:10.1126/science.193.4252.453

Hoang PNT, Michael TP, Gilbert S, Chu P, Motley ST, Appenroth KJ, Schubert I, Lam E. 2018. Generating a high-confidence reference genome map of the greater duckweed by integration of cytogenomic, optical mapping, and Oxford nanopore technologies. Plant J 96: 670-684. doi: $10.1111 /$ tpj. 14049

Hoang PTN, Schubert V, Meister A, Fuchs J, Schubert I. 2019. Variation in genome size, cell and nucleus volume, chromosome number and rDNA loci among duckweeds. Sci Rep 9: 3234. doi:10.1038/s41598019-39332-w

Hoff KJ, Lange S, Lomsadze A, Borodovsky M, Stanke M. 2016. BRAKER1: unsupervised RNA-Seq-based genome annotation with geneMark-ET and AUGUSTUS. Bioinformatics 32: 767-769. doi:10.1093/bioinfor matics/btv661

Hoff KJ, Lomsadze A, Borodovsky M, Stanke M. 2019. Whole-genome annotation with BRAKER. Methods Mol Biol 1962: 65-95. doi:10.1007/978-14939-9173-0_5

Holt C, Yandell M. 2011. MAKER2: an annotation pipeline and genome-database management tool for second-generation genome projects. $B M C$ Bioinformatics 12: 491. doi:10.1186/1471-2105-12-491

Hori K, Ogiso-Tanaka E, Matsubara K, Yamanouchi U, Ebana K, Yano M. 2013. Hd16, a gene for casein kinase $\mathrm{I}$, is involved in the control of rice flowering time by modulating the day-length response. Plant $J \mathbf{7 6}$ 36-46. doi:10.1111/tpj.12268

Huang H, Alvarez S, Bindbeutel R, Shen Z, Naldrett MJ, Evans BS, Briggs SP, Hicks LM, Kay SA, Nusinow DA. 2016. Identification of evening complex associated proteins in Arabidopsis by affinity purification and mass spectrometry. Mol Cell Proteomics 15: 201-217. doi:10.1074/mcp .M115.054064

Huby E, Napier JA, Baillieul F, Michaelson LV, Dhondt-Cordelier S. 2020. Sphingolipids: towards an integrated view of metabolism during the plant stress response. New Phytol 225: 659-670. doi:10.1111/nph.15997

Huerta-Cepas J, Forslund K, Coelho LP, Szklarczyk D, Jensen LJ, von Mering C, Bork P. 2017. Fast genome-wide functional annotation through orthology assignment by eggNOG-mapper. Mol Biol Evol 34: 21152122. doi:10.1093/molbev/msx148

Hughes ME, Hogenesch JB, Kornacker K. 2010. JTK_CYCLE: an efficient nonparametric algorithm for detecting rhythmic components in genome-scale data sets. I Biol Rhythms 25: 372-380. doi:10.1177/ 0748730410379711

Ibarra-Laclette E, Lyons E, Hernández-Guzmán G, Pérez-Torres CA, Carretero-Paulet L, Chang TH, Lan T, Welch AJ, Juárez MJA, Simpson J, et al. 2013. Architecture and evolution of a minute plant genome. Nature 498: 94-98. doi:10.1038/nature12132

Jiang H, Lei R, Ding SW, Zhu S. 2014. Skewer: a fast and accurate adapter trimmer for next-generation sequencing paired-end reads. $B M C$ Bioinformatics 15: 182. doi:10.1186/1471-2105-15-182

Johnson KL, Kibble NAJ, Bacic A, Schultz CJ. 2011. A fasciclin-like arabinogalactan-protein (FLA) mutant of Arabidopsis thaliana, fla1, shows defects in shoot regeneration. PLoS One 6: e25154. doi:10.1371/journal pone. 0025154

Jones JDG, Dangl JL. 2006. The plant immune system. Nature 444: 323-329. doi:10.1038/nature05286

Jones P, Binns D, Chang HY, Fraser M, Li W, McAnulla C, McWilliam H, Maslen J, Mitchell A, Nuka G, et al. 2014. Interproscan 5: genome-scale protein function classification. Bioinformatics 30: 1236-1240. doi:10 .1093/bioinformatics/btu031

Jun J, Fiume E, Roeder AHK, Meng L, Sharma VK, Osmont KS, Baker C, Ha CM, Meyerowitz EM, Feldman LJ, et al. 2010. Comprehensive analysis of CLE polypeptide signaling gene expression and overexpression activity in Arabidopsis. Plant Physiol 154: 1721-1736. doi:10.1104/pp.110 .163683

Katoh K, Standley DM. 2016. A simple method to control over-alignment in the MAFFT multiple sequence alignment program. Bioinformatics 32: 1933-1942. doi:10.1093/bioinformatics/btw108

Kawakatsu T, Huang SSC, Jupe F, Sasaki E, Schmitz RJ, Urich MA, Castanon R, Nery JR, Barragan C, He Y, et al. 2016. Epigenomic diversity in a global collection of Arabidopsis thaliana accessions. Cell 166: 492-505. doi:10 .1016/j.cell.2016.06.044

Kim D, Langmead B, Salzberg SL. 2015. HISAT: a fast spliced aligner with low memory requirements. Nat Methods 12: 357-360. doi:10.1038/nmeth .3317

König S, Romoth LW, Gerischer L, Stanke M. 2016. Simultaneous gene finding in multiple genomes. Bioinformatics 32: 3388-3395. doi:10.1093/ bioinformatics/btw494

Koren S, Walenz BP, Berlin K, Miller JR, Bergman NH, Phillippy AM. 2017. Canu: scalable and accurate long-read assembly via adaptive $k$-mer weighting and repeat separation. Genome Res 27: 722-736. doi:10 $.1101 /$ gr.215087.116

Lam E, Appenroth KJ, Michael T, Mori K, Fakhoorian T. 2014. Duckweed in bloom: the 2nd International Conference on Duckweed Research and Applications heralds the return of a plant model for plant biology. Plant Mol Biol 84: 737-742. doi:10.1007/s11103-013-0162-9

Landolt E. 1986. Biosystematic investigations in the family of duckweeds, Lemnaceae: the family of Lemnaceae, a monographic study. Vol. 1: Morphology, karyology, ecology, geographic distribution, systematic position, nomenclature, descriptions. Geobotanische Institut ETH, Stiftung Rübel, Zurich.

Langmead B, Salzberg SL. 2012. Fast gapped-read alignment with Bowtie 2. Nat Methods 9: 357-359. doi:10.1038/nmeth.1923

Lemon GD, Posluszny U. 2000. Comparative shoot development and evolution in the Lemnaceae. Int J Plant Sci 161: 733-748. doi:10.1086/ 314298

\section{Genome Research}

www.genome.org 
Li H. 2018. Minimap2: pairwise alignment for nucleotide sequences. Bioinformatics 34: 3094-3100. doi:10.1093/bioinformatics/bty191

Liscum E, Briggs WR. 1995. Mutations in the NPH1 locus of Arabidopsis disrupt the perception of phototropic stimuli. Plant Cell 7: 473-485. doi:10 $.1105 /$ tpc.7.4.473

Lou P, Wu J, Cheng F, Cressman LG, Wang X, McClung CR. 2012. Preferential retention of circadian clock genes during diploidization following whole genome triplication in Brassica rapa. Plant Cell 24: $2415-$ 2426. doi:10.1105/tpc.112.099499

Loudet O, Michael TP, Burger BT, Le Metté C, Mockler TC, Weigel D, Chory J. 2008. A zinc knuckle protein that negatively controls morning-specific growth in Arabidopsis thaliana. Proc Natl Acad Sci 105: 17193-17198. doi:10.1073/pnas.0807264105

Lutz KA, Wang W, Zdepski A, Michael TP. 2011. Isolation and analysis of high quality nuclear DNA with reduced organellar DNA for plant genome sequencing and resequencing. BMC Biotechnol 11: 54. doi:10 .1186/1472-6750-11-54

Ma L, Li G. 2018. FAR1-RELATED SEQUENCE (FRS) and FRS-RELATED FACTOR (FRF) family proteins in Arabidopsis growth and development. Front Plant Sci 9: 692. doi:10.3389/fpls.2018.00692

MacKinnon KJM, Cole BJ, Yu C, Coomey JH, Hartwick NT, Remigereau MS Duffy T, Michael TP, Kay SA, Hazen SP. 2020. Changes in ambient temperature are the prevailing cue in determining Brachypodium distachyon diurnal gene regulation. New Phytol 227: 1709-1724. doi:10.1111/nph .16507

Margulies M, Egholm M, Altman WE, Attiya S, Bader JS, Bemben LA, Berka J, Braverman MS, Chen YJ, Chen Z, et al. 2005. Genome sequencing in microfabricated high-density picolitre reactors. Nature 437: 376-380. doi:10.1038/nature03959

Mathur S, Vyas S, Kapoor S, Tyagi AK. 2011. The mediator complex in plants: structure, phylogeny, and expression profiling of representative genes in a dicot (Arabidopsis) and a monocot (rice) during reproduction and abiotic stress. Plant Physiol 157: 1609-1627. doi:10.1104/pp.111 .188300

McClung CR. 2019. The plant circadian oscillator. Biology (Basel) 8: 14 . doi:10.3390/biology8010014

Michael TP, McClung CR. 2002. Phase-specific circadian clock regulatory elements in Arabidopsis. Plant Physiol 130: 627-638. doi:10.1104/pp .004929

Michael TP, McClung CR. 2003. Enhancer trapping reveals widespread circadian clock transcriptional control in Arabidopsis. Plant Physiol 132: 629-639. doi:10.1104/pp.021006

Michael TP, VanBuren R. 2020. Building near-complete plant genomes. Curr Opin Plant Biol 54: 26-33. doi:10.1016/j.pbi.2019.12.009

Michael TP, Salomé PA, Yu HJ, Spencer TR, Sharp EL, McPeek MA, Alonso JM, Ecker JR, McClung CR. 2003. Enhanced fitness conferred by naturally occurring variation in the circadian clock. Science 302: 1049-1053. doi:10.1126/science.1082971

Michael TP, Breton G, Hazen SP, Priest H, Mockler TC, Kay SA, Chory J. 2008a. A morning-specific phytohormone gene expression program underlying rhythmic plant growth. PLoS Biol 6: e225. doi:10.1371/journal .pbio.0060225

Michael TP, Mockler TC, Breton G, McEntee C, Byer A, Trout JD, Hazen SP, Shen R, Priest HD, Sullivan CM, et al. 2008b. Network discovery pipeline elucidates conserved time-of-day-specific cis-regulatory modules. PLOS Genet 4: e14. doi:10.1371/journal.pgen.0040014

Michael TP, Bryant D, Gutierrez R, Borisjuk N, Chu P, Zhang H, Xia J, Zhou J, Peng H, El Baidouri M, et al. 2017. Comprehensive definition of genome features in Spirodela polyrhiza by high-depth physical mapping and short-read DNA sequencing strategies. Plant J 89: 617-635. doi:10 $.1111 /$ tpj. 13400

Mockler TC, Michael TP, Priest HD, Shen R, Sullivan CM, Givan SA, McEntee C, Kay SA, Chory J. 2007. The DIURNAL project: DIURNAL and circadian expression profiling, model-based pattern matching, and promoter analysis. Cold Spring Harb Symp Quant Biol 72: 353-363. doi:10.1101/sqb.2007.72.006

Monnier A, Liverani S, Bouvet R, Jesson B, Smith JQ, Mosser J, Corellou F, Bouget FY. 2010. Orchestrated transcription of biological processes in the marine picoeukaryote Ostreococcus exposed to light/dark cycles. BMC Genomics 11: 192. doi:10.1186/1471-2164-11-192

Motchoulski A, Liscum E. 1999. Arabidopsis NPH3: a NPH1 photoreceptorinteracting protein essential for phototropism. Science 286: 961-964. doi:10.1126/science.286.5441.961

Murakami-Kojima M, Nakamichi N, Yamashino T, Mizuno T. 2002. The APRR3 component of the clock-associated APRR1/TOC1 quintet is phosphorylated by a novel protein kinase belonging to the WNK family, the gene for which is also transcribed rhythmically in Arabidopsis thaliana. Plant Cell Physiol 43: 675-683. doi:10.1093/pcp/pcf084

One Thousand Plant Transcriptomes Initiative. 2019. One thousand plant transcriptomes and the phylogenomics of green plants. Nature 574: 679-685. doi:10.1038/s41586-019-1693-2
Paten B, Earl D, Nguyen N, Diekhans M, Zerbino D, Haussler D. 2011. Cactus: algorithms for genome multiple sequence alignment. Genome Res 21: 1512-1528. doi:10.1101/gr.123356.111

Petricka JJ, Clay NK, Nelson TM. 2008. Vein patterning screens and the defectively organized tributaries mutants in Arabidopsis thaliana. Plant $J$ 56: $251-263$. doi:10.1111/j.1365-313X.2008.03595.x

Pi L, Aichinger E, van der Graaff E, Llavata-Peris CI, Weijers D, Hennig L, Groot E, Laux T. 2015. Organizer-derived WOX5 signal maintains root columella stem cells through chromatin-mediated repression of CDF4 expression. Dev Cell 33: 576-588. doi:10.1016/j.devcel.2015.04.024

Rapparini F, Cohen JD, Slovin JP. 1999. Indole-3-acetic acid biosynthesis in Lemna gibba studied using stable isotope labeled anthranilate and tryptophan. Plant Growth Regul 27: 139-144. doi:10.1023/A :1006191502391

Roppolo D, Boeckmann B, Pfister A, Boutet E, Rubio MC, DénervaudTendon V, Vermeer JEM, Gheyselinck J, Xenarios I, Geldner N. 2014. Functional and evolutionary analysis of the CASPARIAN STRIP MEMBRANE DOMAIN PROTEIN family. Plant Physiol 165: 17091722. doi:10.1104/pp.114.239137

Sarkar AK, Luijten M, Miyashima S, Lenhard M, Hashimoto T, Nakajima K, Scheres B, Heidstra R, Laux T. 2007. Conserved factors regulate signalling in Arabidopsis thaliana shoot and root stem cell organizers. Nature 446: 811-814. doi: $10.1038 /$ nature 05703

Sarris PF, Cevik V, Dagdas G, Jones JDG, Krasileva KV. 2016. Comparative analysis of plant immune receptor architectures uncovers host proteins likely targeted by pathogens. BMC Biol 14: 8. doi:10.1186/s12915-0160228-7

Schatlowski N, Stahl Y, Hohenstatt ML, Goodrich J, Schubert D. 2010. The CURLY LEAF interacting protein BLISTER controls expression of polycomb-group target genes and cellular differentiation of Arabidopsis thaliana. Plant Cell 22: 2291-2305. doi:10.1105/tpc.109.073403

Schindelin J, Arganda-Carreras I, Frise E, Kaynig V, Longair M, Pietzsch T, Preibisch S, Rueden C, Saalfeld S, Schmid B, et al. 2012. Fiji: an opensource platform for biological-image analysis. Nat Methods 9: 676-682. doi:10.1038/nmeth.2019

Schneider M, Lane L, Boutet E, Lieberherr D, Tognolli M, Bougueleret L, Bairoch A. 2009. The UniProtKB/Swiss-Prot knowledgebase and its Plant Proteome Annotation Program. J Proteomics 72: 567-573. doi:10 .1016/j.jprot.2008.11.010

Seluzicki A, Burko Y, Chory J. 2017. Dancing in the dark: darkness as a signal in plants. Plant Cell Environ 40: 2487-2501. doi:10.1111/pce.12900

Simão FA, Waterhouse RM, Ioannidis P, Kriventseva EV, Zdobnov EM. 2015. BUSCO: assessing genome assembly and annotation completeness with single-copy orthologs. Bioinformatics 31: 3210-3212. doi:10.1093/bioin formatics/btv351

Singh B, Sharma RA. 2015. Plant terpenes: defense responses, phylogenetic analysis, regulation and clinical applications. 3 Biotech 5: 129-151. doi:10.1007/s13205-014-0220-2

Singh R, Ong-Abdullah M, Low ETL, Manaf MAA, Rosli R, Nookiah R, Ooi LCL, Ooi SE, Chan KL, Halim MA, et al. 2013. Oil palm genome sequence reveals divergence of interfertile species in old and new worlds. Nature 500: 335-339. doi:10.1038/nature12309

Smit AFA, Hubley R. 2008-2015. RepeatModeler Open-1.0. http://www .repeatmasker.org.

Smit AFA, Hubley R, Green P. 2015. RepeatMasker Open-4.0. 2013-2015. http://www.repeatmasker.org.

Sree KS, Maheshwari SC, Boka K, Khurana JP, Keresztes Á, Appenroth KJ. 2015a. The duckweed Wolffia microscopica: a unique aquatic monocot. Flora Morphol Distrib Funct Ecol Plants 210: 31-39. doi:10.1016/j.flora .2014 .10 .006

Sree KS, Sudakaran S, Appenroth KJ. 2015b. How fast can angiosperms grow? Species and clonal diversity of growth rates in the genus Wolffia (Lemnaceae). Acta Physiol Plant 37: 204. doi:10.1007/s11738-0151951-3

Stanke M, Schöffmann O, Morgenstern B, Waack S. 2006. Gene prediction in eukaryotes with a generalized hidden Markov model that uses hints from external sources. BMC Bioinformatics 7: 62. doi:10.1186/14712105-7-62

Stanke M, Diekhans M, Baertsch R, Haussler D. 2008. Using native and syntenically mapped cDNA alignments to improve de novo gene finding. Bioinformatics 24: 637-644. doi:10.1093/bioinformatics/btn013

Steuernagel B, Witek K, Krattinger SG, Ramirez-Gonzalez RH, Schoonbeek HJ, Yu G, Baggs E, Witek A, Yadav I, Krasileva KV, et al. 2020. The NLR-annotator tool enables annotation of the intracellular immune receptor repertoire. Plant Physiol 183: 468-482. doi:10.1104/pp.19.01273

Sun G, Xu Y, Liu H, Sun T, Zhang J, Hettenhausen C, Shen G, Qi J, Qin Y, Li J, et al. 2018. Large-scale gene losses underlie the genome evolution of parasitic plant Cuscuta australis. Nat Commun 9: 2683. doi:10.1038/ s41467-018-04721-8 
Supek F, Bošnjak M, Škunca N, Šmuc T. 2011. REVIGO summarizes and visualizes long lists of gene ontology terms. PLoS One 6: e21800. doi:10 .1371/journal.pone.0021800

Trapnell C, Roberts A, Goff L, Pertea G, Kim D, Kelley DR, Pimentel H, Salzberg SL, Rinn JL, Pachter L. 2012. Differential gene and transcript expression analysis of RNA-seq experiments with topHat and cufflinks. Nat Protoc 7: 562-578. doi:10.1038/nprot.2012.016

UniProt Consortium. 2019. UniProt: a worldwide hub of protein knowledge. Nucleic Acids Res 47: D506-D515. doi:10.1093/nar/gky1049

Van Bel M, Diels T, Vancaester E, Kreft L, Botzki A, Van de Peer Y, Coppens F, Vandepoele K. 2018. PLAZA 4.0: an integrative resource for functional, evolutionary and comparative plant genomics. Nucleic Acids Res 46: D1190-D1196. doi:10.1093/nar/gkx1002

Vandenbrink JP, Kiss JZ, Herranz R, Medina FJ. 2014. Light and gravity signals synergize in modulating plant development. Front Plant Sci 5: 563. doi:10.3389/fpls.2014.00563

van Wersch S, Li X. 2019. Stronger when together: clustering of plant NLR disease resistance genes. Trends Plant Sci 24: 688-699. doi:10.1016/j .tplants.2019.05.005

Vaser R, Sović I, Nagarajan N, Šikić M. 2017. Fast and accurate de novo genome assembly from long uncorrected reads. Genome Res 27: 737746. doi:10.1101/gr.214270.116

Vogel A, Schwacke R, Denton AK, Usadel B, Hollmann J, Fischer K, Bolger A, Schmidt MH-W, Bolger ME, Gundlach H, et al. 2018. Footprints of parasitism in the genome of the parasitic flowering plant Cuscuta campestris. Nat Commun 9: 2515. doi:10.1038/s41467-018-04344-z

Vurture GW, Sedlazeck FJ, Nattestad M, Underwood CJ, Fang H, Gurtowski J, Schatz MC. 2017. Genomescope: fast reference-free genome profiling from short reads. Bioinformatics 33: 2202-2204. doi:10.1093/bioinfor matics/btx153

Wai CM, Weise SE, Ozersky P, Mockler TC, Michael TP, VanBuren R. 2019 Time of day and network reprogramming during drought induced CAM photosynthesis in Sedum album. PLoS Genet 15: e1008209. doi:10.1371/ journal.pgen.1008209

Walker BJ, Abeel T, Shea T, Priest M, Abouelliel A, Sakthikumar S, Cuomo CA, Zeng Q, Wortman J, Young SK, et al. 2014. Pilon: an integrated tool for comprehensive microbial variant detection and genome assembly improvement. PLoS One 9: e112963. doi:10.1371/journal.pone 0112963
Wang W, Kerstetter RA, Michael TP. 2011. Evolution of genome size in duckweeds (Lemnaceae). J Bot 2011: 570319. doi:10.1155/2011/570319

Wang W, Haberer G, Gundlach H, Gläßer C, Nussbaumer T, Luo MC, Lomsadze A, Borodovsky M, Kerstetter RA, Shanklin J, et al. 2014. The Spirodela polyrhiza genome reveals insights into its neotenous reduction fast growth and aquatic lifestyle. Nat Commun 5: 3311. doi:10.1038/ ncomms 4311

Wick RR, Schultz MB, Zobel J, Holt KE. 2015. Bandage: interactive visualization of de novo genome assemblies. Bioinformatics 31: 3350-3352. doi:10 .1093/bioinformatics/btv383

Willemsen V, Bauch M, Bennett T, Campilho A, Wolkenfelt H, Xu J Haseloff J, Scheres B. 2008. The NAC domain transcription factors FEZ and SOMBRERO control the orientation of cell division plane in Arabidopsis root stem cells. Dev Cell 15: 913-922. doi:10.1016/j.devcel 2008.09.019

Yoshihara T, Spalding EP. 2017. LAZY genes mediate the effects of gravity on auxin gradients and plant architecture. Plant Physiol 175: 959-969. doi:10.1104/pp.17.00942

Zdepski A, Wang W, Priest HD, Ali F, Alam M, Mockler TC, Michael TP. 2008. Conserved daily transcriptional programs in Carica papaya. Trop Plant Biol 1: 236-245. doi:10.1007/s12042-008-9020-3

Zhang S, Li C, Zhou Y, Wang X, Li H, Feng Z, Chen H, Qin G, Jin D, Terzaghi W, et al. 2018. TANDEM ZINC-FINGER/PLUS3 is a key component of phytochrome A signaling. Plant Cell 30: 835-852. doi:10.1105/tpc.17 .00677

Zhao H, Wu D, Kong F, Lin K, Zhang H, Li G. 2016. The Arabidopsis thaliana nuclear factor Y transcription factors. Front Plant Sci 7: 2045. doi:10 3389/fpls.2016.02045

Ziegler P, Adelmann K, Zimmer S, Schmidt C, Appenroth KJ. 2015. Relative in vitro growth rates of duckweeds (Lemnaceae) - the most rapidly growing higher plants. Plant Biol 17 Suppl 1: $33-41$. doi:10.1111/plb.12184

Zones JM, Blaby IK, Merchant SS, Umen JG. 2015. High-resolution profiling of a synchronized diurnal transcriptome from Chlamydomonas reinhardtii reveals continuous cell and metabolic differentiation. Plant Cell 27: 2743-2769. doi:10.1105/tpc.15.00498

Received May 25, 2020; accepted in revised form December 16, 2020. 


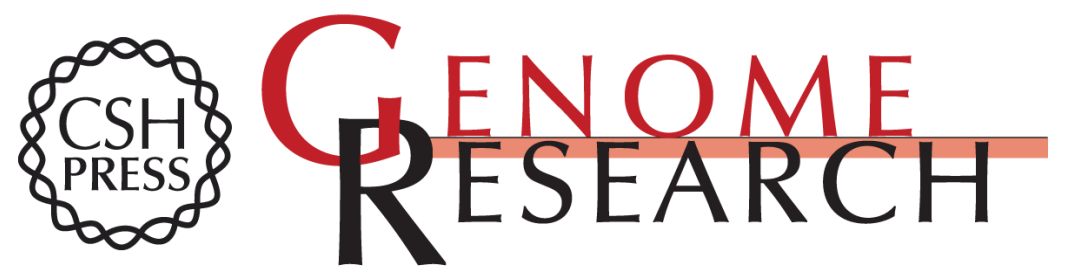

\section{Genome and time-of-day transcriptome of Wolffia australiana link morphological minimization with gene loss and less growth control}

Todd P. Michael, Evan Ernst, Nolan Hartwick, et al.

Genome Res. 2021 31: 225-238 originally published online December 23, 2020

Access the most recent version at doi:10.1101/gr.266429.120

Supplemental Material

References

Open Access

Creative Commons License

Email Alerting Service
http://genome.cshlp.org/content/suppl/2021/01/19/gr.266429.120.DC1

This article cites 113 articles, 29 of which can be accessed free at: http://genome.cshlp.org/content/31/2/225.full.html\#ref-list-1

Freely available online through the Genome Research Open Access option.

This article, published in Genome Research, is available under a Creative Commons License (Attribution-NonCommercial 4.0 International), as described at http://creativecommons.org/licenses/by-nc/4.0/.

Receive free email alerts when new articles cite this article - sign up in the box at the top right corner of the article or click here.

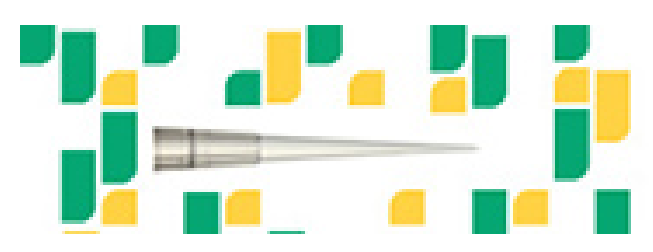

Focused on your science.

Jコగ

SCIENTIFIC

suos or seisnes

To subscribe to Genome Research go to:

https://genome.cshlp.org/subscriptions 